\title{
STABILITY ANALYSIS OF TURING PATTERNS GENERATED BY THE SCHNAKENBERG MODEL
}

\author{
DAVID IRON, JUNCHENG WEI, AND MATTHIAS WINTER
}

ABstraCt. We consider the following Schnakenberg model on the interval $(-1,1)$ :

$$
\begin{cases}u_{t}=D_{1} u^{\prime \prime}-u+v u^{2} & \text { in }(-1,1), \\ v_{t}=D_{2} v^{\prime \prime}+B-v u^{2} & \text { in }(-1,1), \\ u^{\prime}(-1)=u^{\prime}(1)=v^{\prime}(-1)=v^{\prime}(1)=0, & \end{cases}
$$

where

$$
D_{1}>0, \quad D_{2}>0, \quad B>0 .
$$

We rigorously show that the stability of symmetric $N$-peaked steadystates can be reduced to computing two matrices in terms of the diffusion coefficients $D_{1}, D_{2}$ and the number $N$ of peaks. These matrices and their spectra are calculated explicitly and sharp conditions for linear stability are derived. The results are verified by some numerical simulations.

\section{INTRODUCTION}

Since the work of Turing [18] in 1952, a lot of models have been established and investigated to explore the so-called Turing instability.

One of the most interesting models in biological pattern formation is the Schnakenberg model [16] on a one-dimensional interval, which can be stated as follows:

$$
\begin{cases}u_{t}=D_{1} u^{\prime \prime}-u+v u^{2} & \text { in }(-1,1), \\ v_{t}=D_{2} v^{\prime \prime}+B-v u^{2} & \text { in }(-1,1), \\ u^{\prime}(-1)=u^{\prime}(1)=v^{\prime}(-1)=v^{\prime}(1)=0, & \end{cases}
$$

where $D_{1}>0, D_{2}>0, B>0$ are positive constants. Substituting

$$
u=\eta^{-1} \hat{u}, \quad v=\eta \hat{v}
$$

1991 Mathematics Subject Classification. Primary 35B40, 35B45; Secondary 35J55, 92C15, 92C40.

Key words and phrases. Turing instability, Symmetric $N$-peaked solutions, Nonlocal eigenvalue problem. 
and dropping hats we obtain the following form of the system

$$
\begin{cases}u_{t}=\epsilon^{2} u^{\prime \prime}-u+v u^{2} & \text { in }(-1,1), \\ v_{t}=D v^{\prime \prime}+\frac{1}{2}-c v u^{2} & \text { in }(-1,1), \\ u^{\prime}(-1)=u^{\prime}(1)=v^{\prime}(-1)=v^{\prime}(1)=0, & \end{cases}
$$

if we choose

$$
B=\frac{1}{2} \eta
$$

and set

$$
\epsilon^{2}=D_{1}, \quad D=D_{2}, \quad c=\eta^{-2}
$$

Here $c>$ is fixed. The Schnakenberg model is a prototype Turing system for simple chemical reactions with limit cycle behavior.

Turing's basic idea is as follows: Consider a system of reaction-diffusion equations with very different diffusion coefficients. In the case of the Schnakenberg model this basic condition is $\epsilon^{2}<<D$. Then the homogeneous state $u=v=0$ becomes unstable and stable patterns emerge. For the particular Turing patterns of symmetric $N$-peaked solutions we show the following result: In the singular limit $\epsilon<<1$ there are thresholds

$$
D_{1}>D_{2}>D_{3}>\ldots>D_{N}>\ldots
$$

such that for $D>D_{N}$ the $N$-peaked solution is stable and for $D<D_{N}$ the $N$-peaked solution is unstable. Thus we have established the exact stability threshold. Note in particular that for decreasing $D$ more and more $N$-peaked solutions cross this stability threshold and turn stable.

We note that the Schnakenberg model has been widely studied by analytical and numerical methods. We refer to [10] and the references therein in that paper the Schnakenberg model is posed in a two-dimensional square. By using spatially varying diffusion coefficients the degeneracy of the Turing bifurcation is removed and new phenomena appear including stable subcritical striped patterns, and the possibility that stable stripes lose stability supercritically to give stable spotted patterns.

In the present paper we are concerned with a simple type of Turing pattern: Symmetric $N$-peaked solutions in an interval. (See Figure 1 for the case 


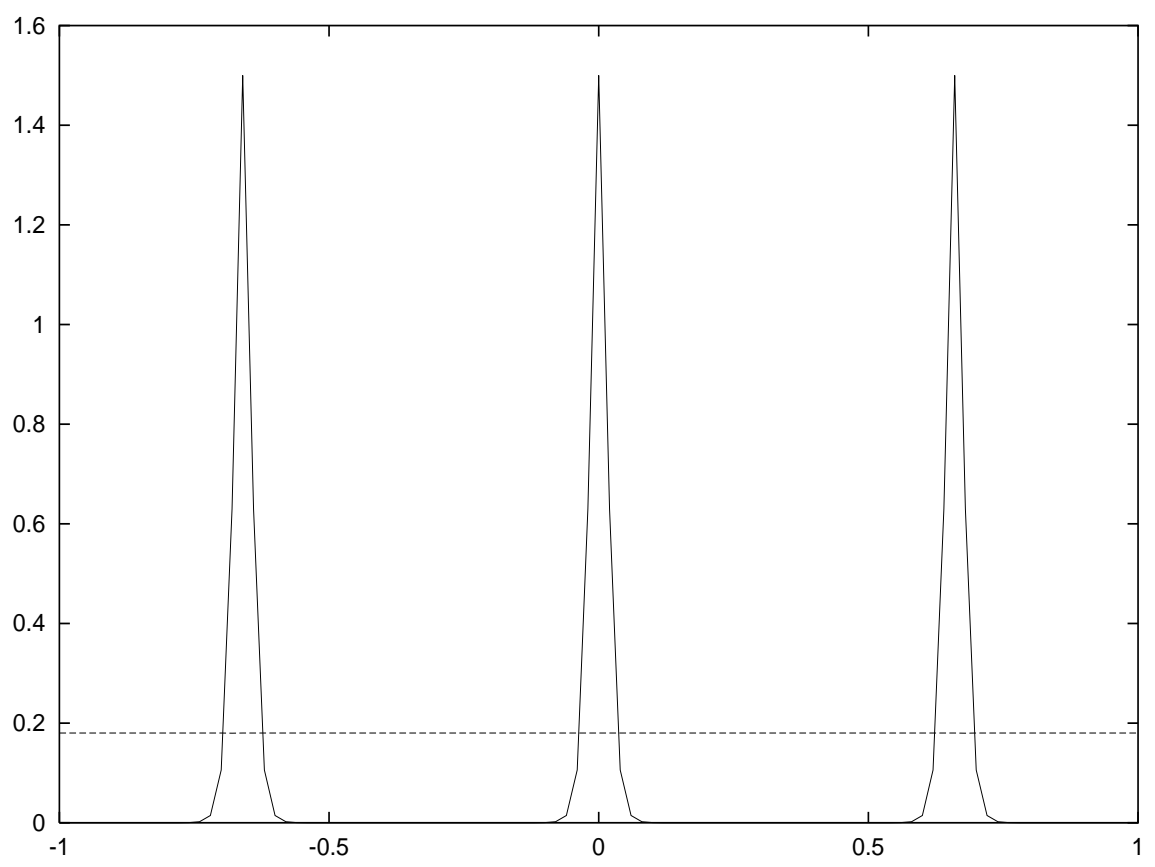

FiguRE 1. Fig. 1: stationary symmetric 3-peaked solutions. Here $D=1, \epsilon=0.01$.

$N=3$.) By symmetric solutions we mean solutions which have a translation symmetry: The solution consists of $N$ peaks on say the interval $\left(-\frac{1}{N}, \frac{1}{N}\right)$ so as to constitute a steady state on $(-1,1)$. Therefore existence which is based on the implicit function theorem follows quite easily from [17]. A proof is included in Appendix A.

What is at the focus of our interest, however, is the question of stability. Here for $\epsilon$ small enough (linear) stability can be explicitly expressed in terms of the diffusion coefficient $D$ and the number $N$ of spikes. Our goal is to provide a rigorous and explicit treatment for the stability of symmetric $N$-peaked solutions in an interval. We emphasize that this analysis is not the classical stability of Turing systems which studies the homogeneous state. Rather we directly explore the stability of our Turing pattern which is far from homogeneity.

We follow the theoretical foundation in [28]. Stability is established by studying the large eigenvalues which tend to a non-zero limit and the small 
eigenvalues which tend to zero in the limit $\epsilon \rightarrow 0$ separately. Large eigenvalues are explored by studying nonlocal eigenvalue problems.

Small eigenvalues are calculated by giving asymptotic expansions with rigorous error estimates. Note that one needs to expand the eigenfunction up to the order $O(\epsilon)$ term. This requires some fine analysis and is done in Section 4.

We believe that our approach can be very useful in the study of other reaction-diffusion systems as well.

A similar analysis for the Gierer-Meinhardt system [5] has been carried out in [28] (proofs) and [9], [19] (matrix calculations). We also refer to [14] for the single-spike case and the survey article [11].

Note also the studies of multi-pulses on the real line [2], [3], [4] where the geometric singular perturbation method is used.

For the Fitzhugh-Nagumo model singular limit eigenvalue analysis has been done in [15].

In higher dimensions, in the shadow system case $(D=\infty)$ the existence of single- or $N$-peaked solutions is established in $[8,7,12,13,20,21,22]$ and other papers. For the Gierer-Meinhardt system in the two-dimensional strong coupling case $(D<\infty)$, the existence of 1-peaked solutions is established in [25], and the stability of $N$-peaked solutions is studied in [26, 27].

Before we state the main results let us find out the right scaling. To this end, set

$$
D=\frac{\hat{D}}{\epsilon}, \quad v=\epsilon \hat{v}, \quad u=\frac{1}{\epsilon} \hat{u} .
$$

Then (1.2) changes to

$$
\begin{cases}\hat{u}_{t}=\epsilon^{2} \hat{u}^{\prime \prime}-\hat{u}+\hat{v} \hat{u}^{2} & \text { in }(-1,1), \\ \epsilon \hat{v}_{t}=\hat{D} \hat{v}^{\prime \prime}+\frac{1}{2}-\frac{c}{\epsilon} \hat{v} \hat{u}^{2} & \text { in }(-1,1), \\ \hat{u}^{\prime}(-1)=\hat{u}^{\prime}(1)=\hat{v}^{\prime}(-1)=\hat{v}^{\prime}(1)=0, & \end{cases}
$$

Let us drop the hats. We obtain the standard form

$$
\begin{cases}u_{t}=\epsilon^{2} u^{\prime \prime}-u+v u^{2} & \text { in }(-1,1), \\ \epsilon v_{t}=D v^{\prime \prime}+\frac{1}{2}-\frac{c}{\epsilon} v u^{2} & \text { in }(-1,1), \\ u^{\prime}(-1)=u^{\prime}(1)=v^{\prime}(-1)=v^{\prime}(1)=0, & \end{cases}
$$


From now on, we work with (1.5) exclusively and we assume that $D>0$ and $c>0$ are constant and independent of $\epsilon$.

Before we state our main results we introduce some notation. Let $L^{2}(-1,1)$ and $H^{2}(-1,1)$ be the usual Lebesgue and Sobolev spaces. With the variable $w$ we denote the unique solution of the following problem:

$$
\begin{cases}w^{\prime \prime}-w+w^{2}=0 & \text { in } R^{1}, \\ w>0, & w(0)=\max _{y \in R} w(y), \\ w(y) \rightarrow 0 & \text { as }|y| \rightarrow \infty\end{cases}
$$

In fact, it is easy to see that $w(y)$ can be written explicitly

$$
w(y)=\frac{3}{2}(\cosh y)^{-2}
$$

The steady-state problem for (1.5) is the following:

$$
\begin{cases}\epsilon^{2} u^{\prime \prime}-u+v u^{2}=0 & \text { in }(-1,1) \\ D v^{\prime \prime}+\frac{1}{2}-\frac{c}{\epsilon} v u^{2}=0 & \text { in }(-1,1) \\ u^{\prime}(-1)=u^{\prime}(1)=v^{\prime}(-1)=v^{\prime}(1)=0, & \end{cases}
$$

We will assume throughout this paper that

$$
D<+\infty, \quad \epsilon<<1
$$

Our first result can be stated as follows:

Theorem 1.1. Assume that $\epsilon<<1$ and $N$ is a positive integer. Then (1.8) admits a solution $\left(u_{\epsilon, N}, v_{\epsilon, N}\right)$ with the following properties:

$$
u_{\epsilon, N}(x)=\xi_{\epsilon}^{-1}\left(\sum_{j=1}^{N} w\left(\frac{x-x_{j}}{\epsilon}\right)+o(1)\right),
$$

where

$$
x_{j}=-1+\frac{2 j-1}{N}, \quad j=1, \ldots, N \text {, }
$$

and $\xi_{\epsilon}$ satisfies

$$
\lim _{\epsilon \rightarrow 0} \xi_{\epsilon}=c N \int_{-\infty}^{\infty} w^{2}:=\xi_{0}
$$

$$
v_{\epsilon, N}\left(x_{j}\right)=\xi_{\epsilon}, \quad j=1, \cdots, N
$$


and $v_{\epsilon, N} \rightarrow v_{0}$, where $v_{0}$ satisfies

$$
\left\{\begin{array}{c}
D \Delta v_{0}+\frac{1}{2}-\frac{1}{N} \sum_{j=1}^{N} \delta_{x_{j}}=0 \\
v_{0}\left(x_{j}\right)=c N \int_{-\infty}^{\infty} w^{2}=\xi_{0}, \quad v_{0}( \pm 1)=0 .
\end{array}\right.
$$

The main purpose of this paper is to study the stability of $\left(u_{\epsilon, N}, v_{\epsilon, N}\right)$. In particular, we shall find an explicit threshold of $D$ - we call it $D_{N}$ - such that below $D_{N},\left(u_{\epsilon, N}, v_{\epsilon, N}\right)$ is linearly stable and above $D_{N},\left(u_{\epsilon, N}, v_{\epsilon, N}\right)$ is linearly unstable. More precisely, we have

Theorem 1.2. Assume that $D$ is finite. For $N \geq 2$, let

$$
D_{N}:=\frac{1}{2 c \int w^{2}} \frac{1}{N^{3}} .
$$

Then for $\epsilon<<1$

(a) $\left(u_{\epsilon, 1}, v_{\epsilon, 1}\right)$ is stable for finite $D$;

(b) for $D<D_{N},\left(u_{\epsilon, N}, v_{\epsilon, N}\right)$ is stable while for $D>D_{N},\left(u_{\epsilon, N}, v_{\epsilon, N}\right)$ is unstable.

Theorem 1.2 is the first result on the stability of multiple-peaked Turing patterns for the Schnakenberg model. It is surprising that an explicit threshold $D_{N}$ can be found. One may ask: What happens at $D \sim D_{N}$ ? It turns out that for $D \sim D_{N}$ asymmetric patterns (namely, Turing patterns consisting of spikes with different heights) appear. For more details, see the recent work of Ward and Wei [19] for the Gierer-Meinhardt system and [24] for the Schnakenberg model.

This paper has the following structure: In Section 2 we study the large eigenvalues of the linearized operator. In Section 3 we begin the study of the small eigenvalues by proving some preliminaries and stating the key lemma, which is Lemma 3.1. In Section 4 we compute the small eigenvalues and prove Lemma 3.1. Section 5 contains some numerical simulations.

Finally, in Appendix A we give an existence proof for steady states and in Appendix B we calculate the matrix $B$ explicitly (which fills a gap left from Section 2). In Appendix $\mathrm{C}$ we compute the matrix $M$ explicitly by calculating the Green's function and their first two derivates, which enables to calculate the expressions in Lemma 3.1 in terms of the diffusion coefficient and the number $N$ of peaks only. 
We use the notation e.s.t to denote an exponentially small term of order $O\left(e^{-d / \epsilon}\right)$ for some $d>0$ in the corresponding norm. By $C$ we denote a generic constant which may change from line to line.

Acknowledgements: The work of JW is supported by an Earmarked Grant of RGC of Hong Kong. MW thanks the Department of Mathematics at CUHK for their kind hospitality. We thank Professor M. J. Ward for valuable discussions.

\section{Stability Analysis: Large Eigenvalues}

In this section, we consider the large eigenvalues of the associated linearized eigenvalue problem.

Let $\left(u_{\epsilon, N}, v_{\epsilon, N}\right)$ be the symmetric $N$-peaked solution constructed in Theorem 1.1 (which is proved in Appendix A). Thus we have by Theorem 1.1 that

$$
u_{\epsilon, N}=\xi_{\epsilon}^{-1} \sum_{j=1}^{N} w\left(\frac{x-x_{j}}{\epsilon}\right), \quad v_{\epsilon, N}\left(x_{j}\right)=\xi_{\epsilon}, \quad j=1, \ldots, N,
$$

where $x_{j}, j=1, \ldots, N$ are the $N$-peaked points given by (1.11).

We linearize (1.5) at $\left(u_{\epsilon, N}, v_{\epsilon, N}\right)$. For simplicity, we drop the index $N$. The eigenvalue problem becomes

$$
\left\{\begin{array}{c}
\epsilon^{2} \phi_{\epsilon}^{\prime \prime}-\phi_{\epsilon}+2 u_{\epsilon} v_{\epsilon} \phi_{\epsilon}+\psi_{\epsilon} u_{\epsilon}^{2}=\lambda_{\epsilon} \phi_{\epsilon}, \\
D \psi_{\epsilon}^{\prime \prime}-\frac{c}{\epsilon} \psi_{\epsilon} u_{\epsilon}^{2}-\frac{2 c}{\epsilon} v_{\epsilon} u_{\epsilon} \phi_{\epsilon}=\epsilon \lambda_{\epsilon} \psi_{\epsilon} .
\end{array}\right.
$$

Here $\lambda_{\epsilon}$ is some complex number and

$$
\phi_{\epsilon}^{\prime}( \pm 1)=\psi_{\epsilon}^{\prime}( \pm 1)=0 .
$$

We consider two cases: The large eigenvalue case with $\lambda_{\epsilon} \rightarrow \lambda_{0} \neq 0$ and the small eigenvalue case, $\lambda_{\epsilon} \rightarrow 0$.

The second case is more involved. We will analyze that case in the next two sections.

Let us now assume that $\lambda_{\epsilon} \rightarrow \lambda_{0}$, where $\lambda_{0}$ is some complex number. 
Let $\chi: R \rightarrow[0,1]$ be a smooth cut-off function such that $\chi(x)=1$ for $|x|<\frac{1}{4 N}$ and $\chi(x)=0$ for $|x|>\frac{1}{2 N}$. Set

$$
\phi_{\epsilon, j}(y)=\phi_{\epsilon}(x) \chi\left(x-x_{j}\right), \quad x=x_{j}+\epsilon y .
$$

Then it is easy to see that from the equation for $\phi_{\epsilon}$

$$
\phi_{\epsilon}(y)=\sum_{j=1}^{N} \phi_{\epsilon, j}(y)+\text { e.s.t. }
$$

in $H^{2}\left(-\frac{1}{\epsilon}, \frac{1}{\epsilon}\right)$.

We assume that

$$
\epsilon^{-1} \int_{-1}^{1} \phi_{\epsilon}^{2}(x) d x \leq C
$$

Hence

$$
\int_{-\frac{1}{2 \epsilon N}}^{\frac{1}{2 \epsilon N}} \phi_{\epsilon, j}^{2}(y) d y \leq C .
$$

Assume that (after a standard extension from $\left(-\frac{1}{\epsilon}, \frac{1}{\epsilon}\right)$ to the real line, see for example [6])

$$
\phi_{\epsilon, j} \rightarrow \phi_{j}(y) \quad \text { in } L^{2}(R)
$$

Now using (2.1) and the equation for $\psi_{\epsilon}$, we have as $\epsilon \rightarrow 0, \psi_{\epsilon} \rightarrow \psi_{0}$, and $\psi_{0}$ satisfies

$$
D \psi_{0}^{\prime \prime}-\gamma \psi_{0} \sum_{j=1}^{N} \delta_{x_{j}}-2 c \sum_{j=1}^{N}\left(\int_{R} w \phi_{j}\right) \delta_{x_{j}}=0
$$

where

$$
\gamma=\frac{c \int_{R} w^{2}}{\xi_{0}^{2}}
$$

Let

$$
\eta=\left(\begin{array}{c}
\eta_{1} \\
\vdots \\
\eta_{N}
\end{array}\right):=\left(\begin{array}{c}
\psi_{0}\left(x_{1}\right) \\
\vdots \\
\psi_{0}\left(x_{N}\right)
\end{array}\right), \quad \Phi=\left(\begin{array}{c}
\phi_{1} \\
\vdots \\
\phi_{N}
\end{array}\right)
$$

In Appendix B, we shall show that the following relations hold:

$$
\left(\frac{D N}{2} K-\gamma I\right) \eta=2 c \int w \Phi
$$


where $K$ is a symmetric matrix given by

$$
K=\left(\begin{array}{lllll}
-1 & 1 & & & 0 \\
1 & -2 & \ddots & & \\
& \ddots & \ddots & \ddots & \\
& & \ddots & -2 & 1 \\
0 & & & 1 & 1
\end{array}\right) .
$$

Thus

$$
\eta=2 c\left(\frac{D N}{2} K-\gamma I\right)^{-1} \int w \Phi
$$

Substituting (2.12) into (2.2), we obtain

$$
\Phi^{\prime \prime}-\Phi+2 w \Phi+2 \frac{c}{\xi_{0}^{2}}\left(\frac{D N}{2} K-\gamma I\right)^{-1}\left(\int_{R} w \Phi\right) w^{2}=\lambda_{0} \Phi .
$$

Set

$$
\mathcal{B}=\gamma\left(\gamma I-\frac{D N}{2} K\right)^{-1}
$$

Then we obtain a system of nonlocal eigenvalue problems

$$
\Phi^{\prime \prime}-\Phi+2 w \Phi-2 \frac{\int_{R} w \mathcal{B} \Phi}{\int_{R} w^{2}} w^{2}=\lambda_{0} \Phi .
$$

The eigenvalues of $\mathcal{B}$ can be computed explicitly. From Appendix B, we have that

Proposition 2.1. The eigenvalues of $\mathcal{B}$ are given by

$$
b_{j}=\left(1-\frac{D N}{\gamma}\left(\cos \frac{\pi(j-1)}{N}-1\right)\right)^{-1}, \quad j=1, \ldots, N
$$

and the corresponding eigenvectors are given by

$$
\begin{gathered}
\mathbf{q}_{1}=\frac{1}{\sqrt{N}}(1, \ldots, 1), \\
\mathbf{q}_{j}=\left(q_{j, 1}, \ldots, q_{j, l}, \ldots, q_{j, N}\right)^{T}, \quad j=2, \ldots, N,
\end{gathered}
$$

where

$$
q_{j, l}=\frac{1}{\sqrt{N}} \cos \frac{\pi(j-1)}{N}\left(l-\frac{1}{2}\right), \quad j=2, \ldots, N, l=1, \ldots, N .
$$


From (2.15) and Proposition 2.1, we see that the large eigenvalues are reduced to the following nonlocal eigenvalue problem (NLEP):

$$
\phi^{\prime \prime}-\phi+2 w \phi-2 b_{j} \frac{\int w \phi}{\int w^{2}} w^{2}=\lambda \phi, \quad j=1, \ldots, N .
$$

Let us recall the following key lemma

Lemma 2.2. [23]: Consider the nonlocal eigenvalue problem

$$
\phi^{\prime \prime}-\phi+2 w \phi-\alpha \frac{\int_{R} w \phi}{\int_{R} w^{2}} w^{2}=\lambda \phi .
$$

(1) If $\alpha<1$, then there is a positive eigenvalue to (2.18).

(2) If $\alpha>1$ then for any nonzero eigenvalue $\alpha$ of (2.18), we have

$$
\operatorname{Re}(\lambda)<0
$$

(3) If $\alpha \neq 1$ and $\lambda=0$, then

$$
\phi=c_{0} w^{\prime}
$$

for some constant $c_{0}$, where $w$ is defined in (1.6).

From Lemma 2.2, we see that the critical threshold for the stability of large eigenvalues is

$$
2 \min _{j=1, \ldots, N} b_{j}>1
$$

which is equivalent to $2 b_{N}>1$, i.e.

$$
2>1-\frac{D N}{\gamma}\left(\cos \frac{\pi(N-1}{N}-1\right)
$$

and thus

$$
D<D_{N}^{1}:=\frac{1}{c N^{3} \int w^{2}\left(1+\cos \frac{\pi}{N}\right)} .
$$

On the other hand, if $D>D_{N}^{1}$, by Lemma (2.2), there exists a positive eigenvalue $\lambda_{0}$ to (2.17) and by perturbation, for $\epsilon$ small, there is a positive eigenvalue for (2.2). (See [1] for related argument.)

In summary, we have arrived at the following proposition:

Proposition 2.3. Let $\lambda_{\epsilon} \rightarrow \lambda_{0} \neq 0$ be an eigenvalue of (2.2). Then

(1) if $D<D_{N}^{1}, \operatorname{Re}\left(\lambda_{\epsilon}\right)<0$,

(2) if $D>D_{N}^{1}$, there exists a positive $\lambda_{\epsilon}$. 
This finishes the study of large eigenvalues.

At the end of this section, we study the following linear operator, which will be useful later. Let

$$
\Phi=\left(\begin{array}{c}
\phi_{1} \\
\phi_{2} \\
\vdots \\
\phi_{N}
\end{array}\right) \in\left(H^{2}(R)\right)^{N}
$$

and

$$
L_{0} \Phi=\Phi^{\prime \prime}-\Phi+2 w \Phi
$$

Let

$$
L \Phi:=\Phi^{\prime \prime}-\Phi+2 w \Phi-2 \frac{\int w \mathcal{B} \Phi}{\int w^{2}} w^{2} .
$$

It is easy to see that the conjugate operator $L^{*}$ is given by

$$
L^{*} \Psi=\Psi^{\prime \prime}-\Psi+2 w \Psi-2 \frac{\int w^{2} \mathcal{B}^{T} \Psi}{\int w^{2}} w
$$

where

$$
\Psi=\left(\begin{array}{c}
\psi_{1} \\
\psi_{2} \\
\vdots \\
\psi_{N}
\end{array}\right) \in\left(H^{2}(R)\right)^{N}
$$

We obtain the following

Lemma 2.4. Assume that $2 b_{j} \neq 1$. Then

$$
\operatorname{Ker}(L)=\left(X_{0}\right)^{N},
$$

where

$$
X_{0}=\operatorname{span}\left\{w^{\prime}(y)\right\},
$$

$w$ is defined in (1.6), and

$$
\operatorname{Ker}\left(L^{*}\right)=\left(X_{0}\right)^{N} .
$$

Proof: Let us first prove (2.23). Suppose

$$
L \Phi=0 .
$$

Let us diagonalize $\mathcal{B}$ such that

$$
P^{-1} \mathcal{B P}=J,
$$


where $P$ is an orthogonal matrix and $J$ has diagonal form, i.e.,

$$
J=\left(\begin{array}{cccc}
d_{1} & & & 0 \\
& d_{2} & & \\
& & \ddots & \\
0 & & & d_{N}
\end{array}\right)
$$

with suitable real numbers $b_{j} \quad j=1,2, \ldots, N$.

Defining

$$
\Phi=P \tilde{\Phi}
$$

we have

$$
\tilde{\Phi}^{\prime \prime}-\tilde{\Phi}+2 w \tilde{\Phi}-2 \frac{\int_{R} w J \tilde{\Phi}}{\int w^{2}} w^{2}=0 .
$$

For $l=1,2, \ldots, N$ we look at the $l$-th equation of system $(2.25)$ :

$$
\tilde{\Phi}_{l}^{\prime \prime}-\tilde{\Phi}_{l}+2 w \tilde{\Phi}_{l}-2 b_{l}\left(\int_{R} w\right)^{-1}\left(\int_{R} w \tilde{\Phi}_{l}\right) w^{2}=0 .
$$

By Lemma 2.18 (3), the last equation (2.26) tells us that

$$
\tilde{\Phi}_{l} \in X_{0} .
$$

Continuing in this way for $l=1, \ldots, N$, we have

$$
\tilde{\Phi}_{l} \in X_{0}, l=1, \ldots, N .
$$

(2.23) is thus proved.

To prove $(2.24)$, we proceed in the same way for $L^{*}$.

Using $\sigma(\mathcal{B})=\sigma\left(\mathcal{B}^{T}\right)$ the $l$-th equation of the diagonalized system is as follows:

$$
\begin{gathered}
\tilde{\Psi}_{l}^{\prime \prime}-\tilde{\Psi}_{l}+2 w \tilde{\Psi}_{l} \\
-2 b_{l}\left(\int_{R} w\right)^{-1}\left(\int_{R} w^{2} \tilde{\Psi}_{l}\right) w=0 .
\end{gathered}
$$

Multiplying (2.29) by $w$ and integrating over the real line, we obtain

$$
\left(1-2 b_{l}\right) \int_{R} w^{2} \tilde{\Psi}_{l}=0
$$

which implies that

$$
\int_{R} w^{2} \tilde{\Psi}_{l}=0
$$

since $2 b_{l} \neq 1$. 
Thus all the non-local terms vanish and we have

$$
L_{0} \tilde{\Psi}_{l}=0, \quad l=1, \ldots, N .
$$

This implies that $\tilde{\Psi}_{l} \in X_{0}$ for $l=1, \ldots, N$.

As a consequence of Lemma 2.4, we have

Lemma 2.5. The operator

$$
L:\left(H^{2}(R)\right)^{N} \rightarrow\left(L^{2}(R)\right)^{N}
$$

is an invertible operator if it is restricted as follows

$$
L:\left(\left(X_{0}\right)^{N}\right)^{\perp} \cap\left(H^{2}(R)\right)^{N} \rightarrow\left(\left(X_{0}\right)^{N}\right)^{\perp} \cap\left(L^{2}(R)\right)^{N} .
$$

Moreover, $L^{-1}$ is bounded.

Proof: This follows from the Fredholm Alternatives Theorem and Lemma 2.4 .

\section{Computation of the small eigenvalues I: PREliminary}

In the next two sections compute the small eigenvalues of the problem (2.2). From Section 2 and Lemma 2.4, if $\lambda_{\epsilon} \rightarrow \lambda_{0}=0$, then

$$
\phi_{\epsilon, j} \rightarrow \phi_{j}
$$

where $L_{0} \Phi=0$. Hence $\phi_{j}=c_{j} w^{\prime}(y)$ for some $c_{j}$. This suggests that the first term in the expansion of $\phi_{\epsilon, j}$ is $a_{j} w^{\prime}(y)$ for some constant $a_{j}$. As we shall prove, the small eigenvalues are of the order $O\left(\epsilon^{2}\right)$. We need to expand the eigenfunction up to the order $O(\epsilon)$-term. Let us define

$$
\tilde{w}_{\epsilon, j}(x)=\chi\left(x-x_{j}\right) u_{\epsilon}(x),
$$

where $\chi(t)$ is defined before (2.4). Then it is easy to see that

$$
u_{\epsilon}(x)=\sum_{j=1}^{N} \tilde{w}_{\epsilon, j}(x)+\text { e.s.t. } \quad \text { in } H^{2}(R) .
$$

Note that

$$
\tilde{w}_{\epsilon, j} \sim \xi_{\epsilon}^{-1} w\left(\frac{x-x_{j}}{\epsilon}\right) \quad \text { in } H_{l o c}^{2}(-1,1)
$$


and $\tilde{w}_{\epsilon, j}(x)$ satisfies

$$
\epsilon^{2} \tilde{w}_{\epsilon, j}-\tilde{w}_{\epsilon, j}+\tilde{w}_{\epsilon, j}^{2} v_{\epsilon}+\text { e.s.t. }=0 \text {. }
$$

Thus $\tilde{w}_{\epsilon, j}^{\prime}:=\frac{d \tilde{w}_{\epsilon, j}}{d x}$ satisfies

$$
\left\{\begin{array}{c}
\epsilon^{2} \tilde{w}_{\epsilon, j}^{\prime}-\tilde{w}_{\epsilon, j}^{\prime}+2 \tilde{w}_{\epsilon, j} v_{\epsilon} \tilde{w}_{\epsilon, j}^{\prime}+\tilde{w}_{\epsilon, j}^{2} v_{\epsilon}^{\prime}+\text { e.s.t. }=0 \\
\tilde{w}_{\epsilon, j}^{\prime}( \pm 1)=0 .
\end{array}\right.
$$

Let us now decompose

$$
\phi_{\epsilon}=\epsilon \sum_{j=1}^{N} a_{j}^{\epsilon} \tilde{w}_{\epsilon, j}^{\prime}+\phi_{\epsilon}^{\perp}
$$

with complex numbers $a_{j}^{\epsilon}$, (the factor $\epsilon$ is for scaling), where

$$
\phi_{\epsilon}^{\perp} \perp \mathcal{K}_{\epsilon, \mathbf{t}^{\epsilon}}=\operatorname{span}\left\{\tilde{w}_{\epsilon, j}^{\prime} \mid j=1, \ldots, N\right\} \subset H^{2}\left(-\frac{1}{\epsilon}, \frac{1}{\epsilon}\right) .
$$

Suppose that $\left\|\phi_{\epsilon}\right\|_{H^{2}\left(\Omega_{\epsilon}\right)}=1$, where $\Omega_{\epsilon}=\left(-\frac{1}{\epsilon}, \frac{1}{\epsilon}\right)$. Then $\left|a_{j}^{\epsilon}\right| \leq C$ since

$$
\left\|\epsilon \tilde{w}_{\epsilon, j}^{\prime}\right\|_{H^{2}\left(-\frac{1}{\epsilon}, \frac{1}{\epsilon}\right)} \geq C>0 \text {. }
$$

Similarly, we can decompose

$$
\psi_{\epsilon}=\epsilon \sum_{j=1}^{N} a_{j}^{\epsilon} \psi_{\epsilon, j}+\psi_{\epsilon}^{\perp},
$$

where $\psi_{\epsilon, j}$ satisfies

$$
\left\{\begin{array}{c}
D \psi_{\epsilon, j}^{\prime \prime}-\frac{c}{\epsilon} \psi_{\epsilon, j} u_{\epsilon}^{2}-2 \frac{c}{\epsilon} v_{\epsilon} u_{\epsilon} \tilde{w}_{\epsilon, j}^{\prime}=\epsilon \lambda_{\epsilon} \psi_{\epsilon, j} \\
\psi_{\epsilon, j}^{\prime}( \pm 1)=0
\end{array}\right.
$$

and $\psi_{\epsilon}^{\perp}$ satisfies

$$
\left\{\begin{array}{c}
D \Delta \psi_{\epsilon}^{\perp}-\frac{c}{\epsilon} \psi_{\epsilon}^{\perp} u_{\epsilon}^{2}-\frac{2 c}{\epsilon} v_{\epsilon} u_{\epsilon} \phi_{\epsilon}^{\perp}=\epsilon \lambda_{\epsilon} \psi_{\epsilon}^{\perp} \\
\left(\psi_{\epsilon}^{\perp}\right)^{\prime}( \pm 1)=0 .
\end{array}\right.
$$

Substituting the decompositions of $\phi_{\epsilon}$ and $\psi_{\epsilon}$ into (2.2) and using (3.4) we have

$$
\begin{gathered}
\epsilon \sum_{j=1}^{N} a_{j}^{\epsilon}\left(u_{\epsilon}^{2} \psi_{\epsilon, j}-\left(\tilde{w}_{\epsilon, j}\right)^{2} v_{\epsilon}^{\prime}\right) \\
+\epsilon^{2}\left(\phi_{\epsilon}^{\perp}\right)^{\prime \prime}-\phi_{\epsilon}^{\perp}+2 u_{\epsilon} v_{\epsilon} \phi_{\epsilon}^{\perp}+u_{\epsilon}^{2} \psi_{\epsilon}^{\perp}-\lambda_{\epsilon} \phi_{\epsilon}^{\perp}+\text { e.s.t. } \\
=\lambda_{\epsilon}\left(\epsilon \sum_{j=1}^{N} a_{j}^{\epsilon} \tilde{w}_{\epsilon, j}^{\prime}\right) .
\end{gathered}
$$


Let us first compute

$$
\begin{gathered}
I_{1}:=\epsilon \sum_{j=1}^{N} a_{j}^{\epsilon}\left(u_{\epsilon}^{2} \psi_{\epsilon, j}-\left(\tilde{w}_{\epsilon, j}\right)^{2} v_{\epsilon}^{\prime}\right) \\
=\epsilon \sum_{j=1}^{N} a_{j}^{\epsilon}\left(\left(\tilde{w}_{\epsilon, j}\right)^{2}\left(\psi_{\epsilon, j}-v_{\epsilon}^{\prime}\right)\right)+\epsilon \sum_{j=1}^{N} a_{j}^{\epsilon} \sum_{k \neq j}\left(\tilde{w}_{\epsilon, k}\right)^{2} \psi_{\epsilon, j}+\text { e.s.t. }
\end{gathered}
$$

(by $(3.2)$ )

$$
\begin{gathered}
=\epsilon \sum_{j=1}^{N} a_{j}^{\epsilon}\left(\tilde{w}_{\epsilon, j}\right)^{2}\left(\psi_{\epsilon, j}-v_{\epsilon}^{\prime}\right) \\
+\epsilon \sum_{j=1}^{N} \sum_{k \neq j} a_{k}^{\epsilon} \psi_{\epsilon, k}\left(\tilde{w}_{\epsilon, j}\right)^{2} .
\end{gathered}
$$

We can rewrite $I_{1}$ as follows

$$
I_{1}=\epsilon \sum_{j=1}^{N} \sum_{k=1}^{N} a_{k}^{\epsilon} \tilde{w}_{\epsilon, j}^{2}\left(\psi_{\epsilon, k}-v_{\epsilon}^{\prime} \delta_{j k}\right)+\text { e.s.t. }
$$

Let us also put

$$
\tilde{L}_{\epsilon} \phi_{\epsilon}^{\perp}:=\epsilon^{2} \Delta \phi_{\epsilon}^{\perp}-\phi_{\epsilon}^{\perp}+2 u_{\epsilon} v_{\epsilon} \phi_{\epsilon}^{\perp}+u_{\epsilon}^{2} \psi_{\epsilon}^{\perp}
$$

and

$$
\mathbf{a}_{\epsilon}:=\left(a_{1}^{\epsilon}, \ldots, a_{N}^{\epsilon}\right)^{T}
$$

Multiplying both sides of $(3.9)$ by $\tilde{w}_{\epsilon, l}^{\prime}$ and integrating over $(-1,1)$, we obtain

$$
\begin{gathered}
\text { r.h.s. }=\epsilon \lambda_{\epsilon} \sum_{j=1}^{N} a_{j}^{\epsilon} \int_{-1}^{1} \tilde{w}_{\epsilon, j}^{\prime} \tilde{w}_{\epsilon, l}^{\prime} \\
=\lambda_{\epsilon} a_{l}^{\epsilon} \hat{\xi}_{l}^{-2} \int_{R}\left(w^{\prime}(y)\right)^{2} d y(1+O(\epsilon))+O\left(\lambda_{\epsilon} \epsilon\left|a_{\epsilon}\right|\right)
\end{gathered}
$$

and

$$
\begin{gathered}
\text { l.h.s. }=\left(\epsilon \sum_{j=1}^{N} \sum_{k=1}^{N} a_{k}^{\epsilon} \int_{-1}^{1} \tilde{w}_{\epsilon, j}^{2}\left(\psi_{\epsilon, k}-v_{\epsilon}^{\prime} \delta_{j k}\right) \tilde{w}_{\epsilon, l}^{\prime}\right. \\
\left.-\int_{-1}^{1} \tilde{w}_{\epsilon, l}^{2} v_{\epsilon}^{\prime} \phi_{\epsilon}^{\perp}+\int_{-1}^{1} \psi_{\epsilon}^{\perp} u_{\epsilon}^{2} w_{\epsilon, l}^{\prime}\right)(1+o(1)) \\
=\left(J_{1, l}+J_{2, l}+J_{3, l}\right)(1+o(1)),
\end{gathered}
$$

where $J_{i, l}, i=1,2,3$ are defined by the last equality. 
We define the vectors

$$
\mathbf{J}_{i}=\left(J_{i, 1}, \ldots, J_{i, N}\right)^{T}, \quad i=1,2,3 .
$$

It remains to compute $\mathbf{J}_{1}, \mathbf{J}_{2}$, and $\mathbf{J}_{3}$. To this end, we need to introduce several matrices.

Let $G_{D}(x, z)$ be Green's function of

$$
\begin{cases}D G_{D}^{\prime \prime}(x, z)+\frac{1}{2}-\delta_{z}=0 & \text { in }(-1,1), \\ \int_{-1}^{1} G_{G}(x, z) d x=0, G_{D}^{\prime}(-1, z)=G_{D}^{\prime}(1, z)=0 . & \end{cases}
$$

We can decompose $G_{D}(x, z)$ as follows

$$
G_{D}(x, z)=\frac{1}{2 D}|x-z|+H_{D}(x, z)
$$

where $H_{D}(x, z)$ is the regular part of $G_{D}$.

We define

$$
\mathcal{G}_{D}=\left(G_{D}\left(x_{i}, x_{j}\right)\right)
$$

Let us denote $\frac{\partial}{\partial x_{i}}$ as $\nabla_{x_{i}}$. When $i \neq j$, we can define $\nabla_{x_{i}} G_{D}\left(x_{i}, x_{j}\right)$ in the classical way. When $i=j$, we define

$$
\nabla_{x_{i}} G_{D}\left(x_{i}, x_{i}\right):=\left.\frac{\partial}{\partial x}\right|_{x=x_{i}} H\left(x, x_{i}\right) .
$$

Now the derivative of $\mathcal{G}$ are defined as follows:

$$
\nabla \mathcal{G}_{D}:=\left(\nabla_{x_{i}} G_{D}\left(x_{i}, x_{j}\right)\right) .
$$

Finally the key matrix $\mathcal{M}$ is defined as

$$
\mathcal{M}:=-\frac{N}{2 D} I-\gamma \nabla \mathcal{G}_{D}\left(I+\gamma \mathcal{G}_{D}\right)^{-1}\left(\nabla \mathcal{G}_{D}\right)^{T} .
$$

Then we have the following key lemma.

Lemma 3.1. For $\epsilon$ sufficiently small, we have

$$
\begin{gathered}
\mathbf{J}_{1}=c_{1} \epsilon^{2}\left[-\frac{N}{2 D} I+\gamma \nabla \mathcal{G}_{D}\left(I-\gamma \mathcal{G}_{D}\right)^{-1}\left(\nabla \mathcal{G}_{D}\right)^{T}\right] \mathbf{a}_{\epsilon}+o\left(\epsilon^{2}\right), \\
\mathbf{J}_{2}=o\left(\epsilon^{2}\right),
\end{gathered}
$$


and

$$
\mathbf{J}_{3}=c_{1} \epsilon^{2}\left[-2 \gamma \nabla \mathcal{G}_{D}\left(I-\gamma \mathcal{G}_{D}\right)^{-1}\left(I+\gamma \mathcal{G}_{D}\right)^{-1}\left(\nabla \mathcal{G}_{D}\right)^{T}+\right] \mathbf{a}_{\epsilon}+o\left(\epsilon^{2}\right),
$$

where $c_{1}$ is given by

$$
c_{1}:=\frac{c \int w^{2} \int w^{3}}{3 \xi_{0}^{4}}>0
$$

and $\gamma$ is given by (2.9).

The proof of Lemma 3.1 is delayed to the next section.

Let us now use it to study the small eigenvalues. In fact, note that

$$
\begin{gathered}
\mathbf{J}_{1}+\mathbf{J}_{2}+\mathbf{J}_{3} \\
=c_{1} \epsilon^{2}\left[-\frac{N}{2 D} I-\gamma \mathcal{G}_{D}\left(I-\gamma \mathcal{G}_{D}\right)^{-1}\left(2\left(I+\gamma \mathcal{G}_{D}\right)^{-1}-I\right)\left(\nabla \mathcal{G}_{D}\right)^{T}\right] \mathbf{a}_{\epsilon}+o\left(\epsilon^{2}\right) \\
=c_{1} \epsilon^{2}\left[-\frac{N}{2 D} I-\gamma \nabla \mathcal{G}_{D}\left(I+\gamma \mathcal{G}_{D}\right)^{-1}\left(\nabla \mathcal{G}_{D}\right)^{T}\right] \mathbf{a}_{\epsilon}+o\left(\epsilon^{2}\right) .
\end{gathered}
$$

Now we use l.h.s. to obtain that

$$
\text { l.h.s. }=c_{1} \epsilon^{2} \frac{1}{\xi_{\epsilon}^{2}} \mathcal{M} \mathbf{a}_{\epsilon}+o\left(\epsilon^{2}\right),
$$

where $\mathcal{M}$ is given by (3.19). Comparing with r.h.s., we have

$$
c_{1} \epsilon^{2} \frac{1}{\xi_{\epsilon}^{2}} \mathcal{M} \mathbf{a}_{\epsilon}+o\left(\epsilon^{2}\right)=\lambda_{\epsilon} \frac{1}{\xi_{\epsilon}^{2}} a_{\epsilon} \int_{R}\left(w^{\prime}(y)\right)^{2} d y+O\left(\epsilon \lambda_{\epsilon}\left|\mathbf{a}_{\epsilon}\right|\right) .
$$

Equation (3.26) shows that the small eigenvalues $\lambda_{\epsilon}$ of (2.2) are

$$
\lambda_{\epsilon} \sim \epsilon^{2} c_{2} \sigma(\mathcal{M})
$$

where

$$
c_{2}=\frac{c_{1}}{\int\left(w^{\prime}\right)^{2}}<0
$$

and $\sigma(\mathcal{M})$ is the spectrum of $\mathcal{M}$. Therefore all that remains is to compute the eigenvalues of $\mathcal{M}$. They are difficult to compute and we delay these calculations to Appendix C.

By Appendix C, we have the following lemma on the eigenvalues of $\mathcal{M}$. 
Lemma 3.2. The eigenvalues of $\mathcal{M}$ are given by

$$
m_{1}=-\frac{N}{2 D}, m_{j}=\frac{N}{2 D} \frac{\tan ^{2} \frac{\theta_{j}}{2}\left(1-\frac{\gamma}{2 D N}\right)}{\tan ^{2} \frac{\theta_{j}}{2}-\frac{\gamma}{2 D N} \sec ^{2} \frac{\theta_{j}}{2}}, \quad j=2, \ldots, N
$$

where $\theta_{j}=\frac{\pi(j-1)}{N}, \quad j=2, \ldots, N$.

From Lemma (3.2), we obtain the following main result of this section.

Proposition 3.3. The small eigenvalues of (2.2) are given by

$$
\lambda_{\epsilon, j}=\epsilon^{2} c_{1} m_{j}, j=1, \ldots, N
$$

where $c_{1}$ and $m_{j}$ are given by (3.23) and (3.29), respectively.

Now combining Proposition 2.3 and Proposition 3.3, we can finish the proof of Theorem 1.2.

\section{Proof of Theorem 1.2:}

We first consider the case $N=1$. In this case, $b_{1}=1$ and thus the large eigenvalue is always stable. For the small eigenvalues, $m_{1}=-\frac{1}{2 D}<0$. So the small eigenvalue is stable, too.

Next we assume that $N \geq 2$. Since if $D>D_{N}^{1}$, we have instability of large eigenvalues, we may consider the case $D<D_{N}^{1}$. In this case, it is easy to see that

$$
\tan ^{2} \frac{\theta}{2}-\frac{\gamma}{2 D N} \sec ^{2} \frac{\theta}{2}<0
$$

By Proposition 3.3, we conclude:

If $1-\frac{\gamma}{2 D N}<0$, we have stability, while if $1-\frac{\gamma}{2 D N}>0$, we have instability.

It is easy to compute that $1-\frac{\gamma}{2 D N}=0$ if and only if

$$
D=\frac{1}{2 N^{3} c \int w^{2}}:=D_{N}^{2}
$$

Since $D_{N}^{1}>D_{N}^{2}$, we see that we have the stability of both large and small eigenvalues if and only if $D<D_{N}^{2}$. This finishes the proof of Theorem 1.2. 


\section{Computation of the small eigenvalues II: Proof of Lemma}

\section{1}

In this section, we prove Lemma 3.1.

We remark that

$$
\begin{gathered}
\int_{-1}^{1} \tilde{w}_{\epsilon, j}^{2}\left(\psi_{\epsilon, k}-v_{\epsilon}^{\prime} \delta_{j k}\right) \tilde{w}_{\epsilon, l}^{\prime} \\
=\int_{-1}^{1} \tilde{w}_{\epsilon, l}^{2}\left(\psi_{\epsilon, k}-v_{\epsilon}^{\prime} \delta_{l k}\right) \tilde{w}_{\epsilon, l}+\text { e.s.t. }
\end{gathered}
$$

So we need to study the asymptotic behavior of $\psi_{\epsilon, k}$ near $x_{l}$. Since $\psi_{\epsilon, k}$ satisfies (3.7), we have that

$$
\psi_{\epsilon, k}(x)-\overline{\psi_{\epsilon, k}}=\frac{c}{\epsilon} \int_{-1}^{1} G_{D}(x, z)\left[\psi_{\epsilon, k} u_{\epsilon}^{2}+2 v_{\epsilon} u_{\epsilon} \tilde{w}_{\epsilon, j}^{\prime}\right] d z .
$$

where $\overline{\psi_{\epsilon, k}}=\frac{1}{2} \int_{-1}^{1} \psi_{\epsilon, k}$. Hence we have

$$
\begin{gathered}
\psi_{\epsilon, j}\left(x_{k}\right)-\overline{\psi_{\epsilon, k}}=\frac{c \int w^{2}}{\xi_{\epsilon}^{2}} \sum_{m=1}^{N} G_{D}\left(x_{k}, x_{m}\right) \psi_{\epsilon, j}\left(x_{m}\right) \\
-\frac{c \int w^{2}}{\xi_{\epsilon}} \sum_{m=1}^{N} \nabla_{x_{m}} G_{D}\left(x_{k}, x_{m}\right)+O\left(\epsilon^{2}\right) .
\end{gathered}
$$

On the other hand, integrating (3.7), we obtain

$$
\frac{c \int w^{2}}{\xi_{\epsilon}^{2}} \sum_{m=1}^{N} \psi_{\epsilon, k}\left(x_{m}\right)=O(\epsilon) .
$$

Note that by Appendix $\mathrm{C}$ we have

$$
\sum_{k=1}^{N} \nabla_{x_{k}} G_{D}\left(x_{k}, x_{m}\right)=0, \quad \sum_{k=1}^{N} G_{D}\left(x_{k}, x_{m}\right)=\lambda_{1}
$$

where $\lambda_{1}$ is a constant independent of $m$. (4.2) and (4.3) imply that

$$
\overline{\psi_{\epsilon, j}}=O(\epsilon) \text {. }
$$

Hence

$$
\Psi_{\epsilon, j}=\left(\begin{array}{c}
\psi_{\epsilon, j}\left(x_{1}\right) \\
\vdots \\
\psi_{\epsilon, j}\left(x_{N}\right)
\end{array}\right)=-\left(I-\gamma \mathcal{G}_{D}\right)^{-1}\left(\nabla \mathcal{G}_{D}\right)^{T} \frac{c \int w^{2}}{\xi_{\epsilon}}+O\left(\epsilon^{2}\right) .
$$

From (4.2) we also see that for $l \neq k$

$$
\psi_{\epsilon, k}\left(x_{l}+\epsilon y\right)-\psi_{\epsilon, k}\left(x_{l}\right)
$$




$$
\begin{gathered}
=\left(\frac{c \int w^{2}}{\xi_{\epsilon}^{2}} \sum_{m=1}^{N} \nabla_{x_{l}} G_{D}\left(x_{l}, x_{m}\right) \psi_{\epsilon, k}\left(x_{m}\right)\right. \\
\left.-\frac{c \int w^{2}}{\xi_{\epsilon}} \nabla_{x_{l}} \nabla_{x_{m}} G_{D}\left(x_{k}, x_{m}\right)\right) \epsilon y+O\left(\epsilon^{2}\right) \\
=\frac{c \int w^{2}}{\xi_{\epsilon}^{2}} \sum_{m=1}^{N} \nabla_{x_{l}} G_{D}\left(x_{k}, x_{m}\right) \psi_{\epsilon, k}\left(x_{m}\right) \epsilon y+O\left(y^{2}\right)
\end{gathered}
$$

since

$$
\nabla_{x_{l}} \nabla_{x_{m}} G_{D}\left(x_{k}, x_{m}\right)=0
$$

At $l=k, v_{\epsilon}^{\prime}$ satisfies

$$
\left\{\begin{array}{c}
D\left(v_{\epsilon}^{\prime}\right)^{\prime \prime}-\frac{c}{\epsilon} u_{\epsilon}^{2} v_{\epsilon}^{\prime}-\frac{2 c}{\epsilon} u_{\epsilon} v_{\epsilon} \tilde{w}_{\epsilon, l}^{\prime}=0, \\
v_{\epsilon}^{\prime}\left(x_{l}-\frac{1}{N}\right)=v_{\epsilon}^{\prime}\left(x_{l}+\frac{1}{N}\right)=0 .
\end{array}\right.
$$

Since the Green's function for $D u^{\prime \prime}=\delta_{z},-\frac{1}{N}<x<\frac{1}{N}, u\left(-\frac{1}{N}\right)=u\left(\frac{1}{N}\right)$ is $\frac{1}{2 D}|x-z|+\frac{N}{2 D}\left(x z-\frac{1}{N^{2}}\right)$, we have that $v_{\epsilon}^{\prime}(x)$ satisfies for $x=x_{l} \epsilon+y, z=x_{l}+\epsilon \bar{z}$

$$
\begin{gathered}
v_{\epsilon}^{\prime}\left(x_{l}+\epsilon y\right)=\frac{c}{\epsilon} \int_{-\frac{1}{N}}^{\frac{1}{N}}\left(\frac{1}{2 D}|x-z|+\frac{N}{2 D}\left(x z-\frac{1}{N^{2}}\right)\right)\left(u_{\epsilon}^{2} v_{\epsilon}^{\prime}+2 u_{\epsilon} v_{\epsilon} \tilde{w}_{\epsilon, l}^{\prime}\right) d z \\
=\frac{c}{\epsilon} \int_{-\frac{1}{N \epsilon}}^{\frac{1}{N \epsilon}}\left(\frac{1}{2 D} \epsilon|y-\bar{z}|+\frac{N}{2 D}\left(\epsilon^{2} y \bar{z}\right)\right)\left(u_{\epsilon}^{2} v_{\epsilon}^{\prime}+2 u_{\epsilon} v_{\epsilon} \tilde{w}_{\epsilon, l}^{\prime}\right) \epsilon d \bar{z} \\
=\frac{2 c}{\xi_{\epsilon}} \int_{-\frac{1}{N \epsilon}}^{\frac{1}{N \epsilon}}|y-\bar{z}| w w^{\prime}+\frac{N c}{2 D} \epsilon y \int_{-\infty}^{\infty} \frac{2 w w^{\prime} \bar{z}}{\xi_{\epsilon}} d \bar{z}+O\left(\epsilon^{2}\right) .
\end{gathered}
$$

Similarly

$$
\begin{gathered}
\psi_{\epsilon, l}\left(x_{l}+\epsilon y\right)-\psi_{\epsilon, l}\left(x_{l}\right) \\
=\frac{c}{\epsilon} \int_{-1}^{1}\left[G_{D}\left(x_{l}+\epsilon y, z\right)-G_{D}\left(x_{l}, z\right)\right]\left(\psi_{\epsilon, l} u_{\epsilon}^{2}+2 v_{\epsilon} u_{\epsilon} \tilde{w}_{\epsilon, l}^{\prime}\right) d z \\
=\frac{c}{\epsilon} \int_{-1}^{1}\left[\frac{1}{2 D}\left(\left|x_{l}+\epsilon y-z\right|-\left|x_{l}-z\right|\right)-\frac{\left(x_{l}+\epsilon y\right)^{2}-x_{l}^{2}}{4 D}\right]\left(\psi_{\epsilon, l} u_{\epsilon}^{2}+2 v_{\epsilon} u_{\epsilon} \tilde{w}_{\epsilon, l}^{\prime}\right) d z \\
=\frac{c}{2 D} \epsilon \int_{-\frac{1}{\epsilon}}^{\frac{1}{\epsilon}}(|y-\bar{z}|-|\bar{z}|) \psi_{\epsilon, j}\left(x_{l}\right) \frac{w^{2}}{\xi_{\epsilon}^{2}} d \bar{z} \\
-\frac{c \epsilon x_{l} y}{2 D} \psi_{\epsilon, l}\left(x_{l}\right) \frac{\int w^{2}}{\xi_{\epsilon}^{2}}+\frac{2 c}{\xi_{\epsilon}} \int_{-\infty}^{\infty}|y-\bar{z}| w(\bar{z}) w^{\prime}(\bar{z}) d \bar{z}
\end{gathered}
$$

Hence

$$
\begin{gathered}
\psi_{\epsilon, l}\left(x_{l}+\epsilon y\right)-\psi_{\epsilon, l}\left(x_{l}\right)-\left(v_{\epsilon}^{\prime}\left(x_{l}+\epsilon y\right)\right) \\
=\frac{c}{2 D} \epsilon \int_{-\frac{1}{\epsilon}}^{\frac{1}{\epsilon}}(|y-\bar{z}|-|\bar{z}|) \frac{w^{2}(\bar{z})}{\xi_{\epsilon}^{2}} \psi_{\epsilon, j}\left(x_{l}\right) d \bar{z}+\epsilon y \nabla_{x_{l}} G_{D}\left(x_{l}, x_{l}\right) \psi_{\epsilon, l}\left(x_{l}\right) \frac{c \int w^{2}}{\xi_{\epsilon}^{2}}
\end{gathered}
$$




$$
+\frac{N}{2 D} \epsilon y \frac{c \int w^{2}}{\xi_{\epsilon}}+O\left(\epsilon^{2}\right) .
$$

Combining (4.6) and (4.10), we have

$$
\begin{gathered}
\left(\psi_{\epsilon, k}-v_{\epsilon}^{\prime} \delta_{l k}\right)\left(x_{l}+\epsilon y\right)-\left(\psi_{\epsilon, k}-v_{\epsilon}^{\prime} \delta_{l k}\right)\left(x_{l}\right) \\
=\frac{c}{2 D} \epsilon \int_{-\frac{1}{\epsilon}}^{\frac{1}{\epsilon}}(|y-\bar{z}|-|\bar{z}|) \frac{w^{2}}{\xi_{\epsilon}^{2}} \psi_{\epsilon, j}\left(x_{l}\right) \delta_{l k} \\
+\frac{c \int w^{2}}{\xi_{\epsilon}^{2}} \sum_{m=1}^{N} \nabla_{x_{l}} G_{D}\left(x_{k}, x_{m}\right) \psi_{\epsilon, j}\left(x_{m}\right) \epsilon y \\
+\frac{N}{2 D} \epsilon y \frac{c \int w^{2}}{\xi_{\epsilon}} \delta_{l k}+O\left(\epsilon^{2}\right) .
\end{gathered}
$$

Substituting (4.11) into the computation of $\mathbf{J}_{1}$, we obtain that

$$
\begin{gathered}
\mathbf{J}_{1}=\epsilon^{2} \frac{1}{\xi_{\epsilon}^{3}} c \int_{\infty}^{\infty} y w^{2}(y) w^{\prime}(y) d y \\
{\left[\frac{N}{2 D} \frac{c \int_{-\infty}^{\infty} w^{2}}{\xi_{\epsilon}} I-\frac{c \int w^{2}}{\xi_{\epsilon}^{2}} \nabla \mathcal{G}_{D}\left(I+\gamma \mathcal{G}_{D}\right)^{-1}\left(\nabla \mathcal{G}_{D}\right)^{T} \frac{c \int w^{2}}{\xi_{\epsilon}}+O(\epsilon)\right] \mathbf{a}_{\epsilon}} \\
=\epsilon^{2} \frac{1}{\xi_{\epsilon}^{3}}\left(-\frac{1}{3} \int_{-\infty}^{\infty} w^{3}\right)\left[\frac{N}{2 D} I-\gamma \nabla \mathcal{G}_{D}\left(I-\gamma \mathcal{G}_{D}\right)^{-1}\left(\nabla \mathcal{G}_{D}\right)^{T}+O(\epsilon)\right] \frac{c \int w^{2}}{\xi_{\epsilon}} \mathbf{a} \\
=-\frac{c \epsilon^{2} \int w^{3} \int w^{2}}{3 \xi_{\epsilon}^{4}}\left[\frac{N}{2 D} I-\gamma \nabla \mathcal{G}_{D}\left(I-\gamma \mathcal{G}_{D}\right)^{-1}\left(\nabla \mathcal{G}_{D}\right)^{T}+O(\epsilon)\right] \mathbf{a}_{\epsilon} \\
=c_{1} \epsilon^{2}\left[\nabla^{2} \mathcal{G}_{D}+\gamma \nabla \mathcal{G}_{D}\left(I-\gamma \mathcal{G}_{D}\right)^{-1}\left(\nabla \mathcal{G}_{D}\right)^{T}+O(\epsilon)\right] \mathbf{a}_{\epsilon}
\end{gathered}
$$

which proves the asymptotic expansion for $\mathbf{J}_{1}$.

Next we compute $\mathbf{J}_{2}$ and $\mathbf{J}_{3}$. To this end, we need to study the asymptotic behavior of $\phi_{\epsilon}^{\perp}$. Let

$$
\tilde{\phi}_{\epsilon, j}^{\perp}=\frac{1}{\epsilon} \phi_{\epsilon}^{\perp} \chi\left(x-x_{j}\right) .
$$

Then it is easy to see that

$$
\phi_{\epsilon}^{\perp}=\epsilon \sum_{j=1}^{N} \tilde{\phi}_{\epsilon, j}^{\perp}+O\left(\epsilon^{2}\right) \quad \text { in } H^{2}\left(\Omega_{\epsilon}\right) .
$$

Set

$$
\tilde{\Phi}_{\epsilon}^{\perp}=\left(\begin{array}{c}
\tilde{\phi}_{\epsilon, 1}^{\perp} \\
\vdots \\
\tilde{\phi}_{\epsilon, N}^{\perp}
\end{array}\right) .
$$


By the equation for $\psi_{\epsilon}^{\perp}$ :

$$
D \Delta \psi_{\epsilon}^{\perp}-\frac{c}{\epsilon} u_{\epsilon}^{2} \psi_{\epsilon}^{\perp}-\frac{2 c}{\epsilon} u_{\epsilon} v_{\epsilon} \psi_{\epsilon}^{\perp}=\epsilon \lambda_{\epsilon} \psi_{\epsilon}^{\perp}
$$

we see that

$$
\psi_{\epsilon}^{\perp}\left(x_{i}\right)-\overline{\psi_{\epsilon}^{\perp}}=\frac{c \int w^{2}}{\xi_{\epsilon}^{2}} \sum_{m=1}^{N} G_{D}\left(x_{i}, x_{m}\right) \psi_{\epsilon}^{\perp}\left(x_{m}\right)+2 c \epsilon \sum_{m=1}^{N} G_{D}\left(x_{i}, x_{m}\right) \int w \phi_{\epsilon, m}^{\perp} .
$$

Hence

$$
\Psi_{\epsilon}^{\perp}=\left(\begin{array}{c}
\psi_{\epsilon}^{\perp}\left(x_{1}\right) \\
\vdots \\
\psi_{\epsilon}^{\perp}\left(x_{N}\right)
\end{array}\right)=\left(I-\gamma \mathcal{G}_{D}\right)^{-1}\left(2 c \epsilon \mathcal{G}_{D} \int w \tilde{\phi}_{\epsilon}^{\perp}+\mathbf{e} \overline{\psi_{\epsilon}^{\perp}}\right)
$$

where

$$
\mathbf{e}=\left(\begin{array}{c}
1 \\
\vdots \\
1
\end{array}\right)
$$

Note that

$$
\gamma \bar{\psi}+2 c \epsilon \sum_{m=1}^{N} \int w \tilde{\phi}_{\epsilon, m}^{\perp}=0
$$

Let

$$
E=\left(\begin{array}{cccc}
1 & 1 & \cdots & 1 \\
1 & 1 & \cdots & 1 \\
\vdots & \vdots & \vdots & \vdots \\
1 & 1 & \cdots & 1
\end{array}\right)
$$

Then

$$
\Psi_{\epsilon}^{\perp}=\left(I-\gamma \mathcal{G}_{D}\right)^{-1}\left(2 c \epsilon \mathcal{G}_{D}-\frac{2 c \epsilon}{\gamma} E\right) \int_{R} w \tilde{\Phi}_{\epsilon}^{\perp}
$$

and

$$
\begin{gathered}
\psi_{\epsilon}^{\perp}\left(x_{l}+\epsilon y\right)-\psi_{\epsilon}^{\perp}\left(x_{l}\right) \\
=\epsilon y \gamma \sum_{m=1}^{N} \nabla_{x_{l}} G_{D}\left(x_{l}, x_{m}\right) \psi_{\epsilon}^{\perp}\left(x_{m}\right)+\epsilon^{2} y 2 c \sum_{m=1}^{N} \nabla_{x_{l}} G_{D}\left(x_{l}, x_{m}\right) \int w \phi_{\epsilon, m}^{\perp}
\end{gathered}
$$

By (4.5) we see that as $\epsilon \rightarrow 0$

$$
\tilde{\phi}_{\epsilon, j} \rightarrow \tilde{\phi}_{\epsilon, j}^{\perp}
$$


We introduce the notation

$$
\tilde{\Phi}^{\perp}=\left(\begin{array}{c}
\tilde{\phi}_{1}^{\perp} \\
\vdots \\
\tilde{\phi}_{N}^{\perp}
\end{array}\right)
$$

where $\tilde{\Phi}^{\perp}$ satisfies

$$
\left\{\begin{array}{c}
\left(\tilde{\Phi}^{\perp}\right)^{\prime \prime}-\tilde{\Phi}^{\perp}+2 w \tilde{\Phi}^{\perp}-\frac{2 \int w \mathcal{B} \tilde{\Phi}^{\perp}}{\int w^{2}} w^{2}+\left(I-\gamma \mathcal{G}_{D}\right)^{-1}\left(\nabla \mathcal{G}_{D}\right)^{T} \mathbf{a}^{0} \frac{c \int w^{2}}{\xi_{0}}=0 \\
\tilde{\Phi}^{\perp} \perp\left(X_{0}\right)^{N}
\end{array}\right.
$$

with

$$
\begin{gathered}
\mathcal{B}=-\frac{\int w^{2}}{\xi_{0}}\left(I-\gamma \mathcal{G}_{D}\right)^{-1}\left(c \mathcal{G}_{D}-\frac{c}{\gamma} E\right) \\
=-\left(I-\gamma \mathcal{G}_{D}\right)^{-1}\left(\gamma \mathcal{G}_{D}-E\right) \\
\lim _{\epsilon \rightarrow 0} \mathbf{a}_{\epsilon}=\mathbf{a}^{0} .
\end{gathered}
$$

Hence

$$
\begin{gathered}
\tilde{\Phi}^{\perp}=-(I-2 \mathcal{B})^{-1}\left(I-\gamma \mathcal{G}_{D}\right)^{-1}\left(\nabla \mathcal{G}_{D}\right)^{T} \mathbf{a}^{0} \frac{c \int w^{2}}{\xi_{0}} w, \\
\Psi_{\epsilon}^{\perp}=\left(I-\gamma \mathcal{G}_{D}\right)^{-1}\left(2 c \epsilon \mathcal{G}_{D}-\frac{2 c \epsilon}{\gamma} E\right) \int_{R} w \tilde{\Phi}_{\epsilon}^{\perp} \\
=-\left(I-\gamma \mathcal{G}_{D}\right)^{-1}\left(2 c \epsilon \mathcal{G}_{D}-\frac{2 c \epsilon}{\gamma} E\right) \int w^{2}(I-2 \mathcal{B})^{-1}\left(I-\gamma \mathcal{G}_{D}\right)^{-1}\left(\nabla \mathcal{G}_{D}\right)^{T} \frac{c \int w^{2}}{\xi_{0}}
\end{gathered}
$$

and so we have

$$
\begin{gathered}
\mathbf{J}_{3}=\epsilon^{2} \frac{\int_{R} y w^{2} w^{\prime}}{\xi_{\epsilon}^{3}}\left[\gamma \nabla \mathcal{G}_{D} \psi_{\epsilon}^{\perp}+2 c \nabla \mathcal{G}_{D} \int_{R} w \tilde{\Phi}^{\perp}\right] \\
=\epsilon^{2} \frac{\int y w^{2} w^{\prime}}{\xi_{\epsilon}^{3}} \frac{c \int w^{2}}{\xi_{0}}\left[\gamma \nabla \mathcal{G}_{D}\left(I-\gamma \mathcal{G}_{D}\right)^{-1}\left(2 c G_{D}-\frac{2 c}{\gamma} E\right)+2 c \nabla \mathcal{G}_{D}\right] \int w \tilde{\Phi}^{\perp} \\
=\epsilon^{2} c_{1}\left[\nabla \mathcal{G}_{D}\left(I-\gamma \mathcal{G}_{D}\right)^{-1}\left(2 I-\frac{2}{\gamma} E\right)\right. \\
\left.-(I-2 \mathcal{B})^{-1}\left(I-\gamma \mathcal{G}_{D}\right)^{-1}\left(\nabla \mathcal{G}_{D}\right)^{T}\right] \mathbf{a}^{0}
\end{gathered}
$$

Note that

$$
\begin{gathered}
\mathcal{B}=\left(I-\gamma \mathcal{G}_{D}\right)^{-1}\left(\gamma \mathcal{G}_{D}-E\right) \\
I-2 \mathcal{B}=I+2\left(I-\gamma \mathcal{G}_{D}\right)^{-1}\left(\gamma \mathcal{G}_{D}-E\right) \\
=\left(I-\gamma \mathcal{G}_{D}\right)^{-1}\left(I+\gamma \mathcal{G}_{D}-2 E\right), \\
(I-2 \mathcal{B})^{-1}=\left(I+\gamma \mathcal{G}_{D}-2 E\right)^{-1}\left(I-\gamma \mathcal{G}_{D}\right) .
\end{gathered}
$$


So

$$
\begin{gathered}
\nabla \mathcal{G}_{D}\left(I-\gamma \mathcal{G}_{D}\right)^{-1}\left(I-\frac{E}{\gamma}\right)\left(-(I-2 \mathcal{B})^{-1}\left(I-\gamma \mathcal{G}_{D}\right)^{-1}\right)\left(\nabla \mathcal{G}_{D}\right)^{T} \\
=-\nabla \mathcal{G}_{D}\left(I-\gamma \mathcal{G}_{D}\right)^{-1}\left(I-\frac{E}{\gamma}\right)\left(I+\gamma \mathcal{G}_{D}-2 E\right)^{-1}\left(\nabla \mathcal{G}_{D}\right)^{T}
\end{gathered}
$$

Note that $E\left(\nabla \mathcal{G}_{D}\right)^{T}=0$ and $E \mathcal{G}_{D}=\mathcal{G}_{D} E$. Hence

$$
\left(I+\gamma \mathcal{G}_{D}-2 E\right)^{-1}\left(\nabla \mathcal{G}_{D}\right)^{T}=\left(I+\gamma \mathcal{G}_{D}\right)^{-1} \nabla \mathcal{G}_{D}
$$

and

$$
\mathbf{J}_{3}=c_{1} \epsilon^{2}\left[-2 \nabla \mathcal{G}_{D}\left(I-\gamma \mathcal{G}_{D}\right)^{-1}\left(I+\gamma \mathcal{G}_{D}\right)^{-1}\left(\nabla \mathcal{G}_{D}\right)^{T}\right] \mathbf{a}_{\epsilon}=o\left(\epsilon^{2}\right),
$$

which proves (3.22).

\section{Numerical Simulations}

We have performed some numerical simulations to verify our results. In all our computations, we take $c=1$ and work with the rescaled equation (1.5). By Theorem 1.2, we have

$$
D_{N}=\frac{1}{2 c \int_{R} w^{2} N^{3}}
$$

Since $\int_{R} w^{2}=6$, we can compute

$$
D_{N}=\frac{1}{12 N^{3}}
$$

Let us first consider the dynamics of one-peaked solution first. It is wellknown that the single-interior spike solution is unstable when $D=+\infty$. (See [23].) However, if we take $D<+\infty$, the one-peak solution can become stable. We have computed the dynamics of one-peaked solution when $D=5$ and $\epsilon=0.05$. Figure 2 contains the trajectories of $u$ at different times: $t=50, t=2500, t=50000$.

The trajectory of the center of the spike is given by Figure 3. We see that the trajectory converges to the center.

Next we consider the stability of two-peaked solutions. We start with $D=1, \epsilon=0.006$. By our theory, the critical thereshold for the stability of two-peaked solution is $D_{2}=\frac{1}{96} \sim 0.01$. So it should be unstable and our numerical computation confirms that. See Figure 4. 
Finally, we decrease $D$ to be $D=0.008<D_{2}$. By Theorem 1.2 , the two-peaked solution should be stable. Figure 5 confirms that.

Figure 6 shows that the trajectories of the centers of the two-peaked solution converge to the stationary two-peaked location.

\section{Appendix A. Existence of $N$-peaked solutions}

We prove Theorem 1.1 in this appendix. Since this is similar to that of [17], we shall give a sketch only. The main idea is that we restrict the solution to be symmetric and then apply the implicit function theorem.

To this end, it is enough to consider the following problem

$$
\left\{\begin{array}{cl}
\epsilon^{2} u^{\prime \prime}-u+v u^{2}=0 & \text { in }(-l, l) \\
D v^{\prime \prime}+\frac{1}{2}-\frac{c}{\epsilon} v u^{2}=0 & \text { in }(-l, l) \\
u^{\prime}( \pm l)=v^{\prime}( \pm l)=0 &
\end{array}\right.
$$

where $l=\frac{1}{N}$. We construct symmetric single-peaked solutions to (6.1). Then by pasting $N$ of these solutions together we obtain a symmetric $N$-peaked solution in $(-1,1)$.

Let

$$
H_{s}^{2}\left(-\frac{l}{\epsilon}, \frac{l}{\epsilon}\right)=\left\{u \in H^{2}\left(-\frac{l}{\epsilon}, \frac{l}{\epsilon}\right) \mid u(y)=u(-y), u^{\prime}\left( \pm \frac{\epsilon}{l}\right)=0\right\} .
$$

Fix $u \in H_{s}^{2}$. We can solve for $v$ first: Let $v=T[u]$ be the unique solution of

$$
\left\{\begin{array}{c}
D v^{\prime \prime}+\frac{1}{2}-\frac{c}{\epsilon} v u^{2}=0 \quad \text { in }(-l, l) \\
v^{\prime}( \pm l)=0 .
\end{array}\right.
$$

Then problem (6.1) can be re-written as a single non-local equation;

$$
\left\{\begin{array}{c}
\Delta_{y} u-u+T[u] u^{2}=0 \quad \text { in }\left(-\frac{l}{\epsilon}, \frac{l}{\epsilon}\right), \quad y=\frac{x}{\epsilon}, \\
u \in H_{r}^{2}\left(-\frac{l}{\epsilon}, \frac{l}{\epsilon}\right) .
\end{array}\right.
$$

Recall that

$$
\xi_{0}=c N \int_{\infty}^{\infty} w^{2}
$$

and define

$$
w_{\epsilon}(x)=\frac{1}{\xi_{0}} w\left(\frac{x}{\epsilon}\right) \chi(x),
$$

where $\chi$ is defined before (2.4). Set $u=w_{\epsilon}+\phi_{\epsilon}$. Then we have

$$
\epsilon^{2} \phi_{\epsilon}^{\prime \prime}-\phi_{\epsilon}+2 T\left[w_{\epsilon}\right] w_{\epsilon} \phi_{\epsilon}+T^{\prime}\left[w_{\epsilon}\right]\left(\phi_{\epsilon}\right) w_{\epsilon}^{2}
$$




$$
+T\left[w_{\epsilon}\right] w_{\epsilon}^{2}-\frac{1}{\xi_{0}} w_{\epsilon}^{2}+\text { e.s.t. }+N\left[\phi_{\epsilon}\right]=0
$$

in $H^{2}(-l, l)$, where $N[\phi]$ represents all quadratic and higher-order terms in $\phi_{\epsilon}$. Let

$$
S_{\epsilon}\left[\phi_{\epsilon}\right]=\phi_{\epsilon}^{\prime \prime}-\phi_{\epsilon}+2 T\left[w_{\epsilon}\right] w_{\epsilon} \phi_{\epsilon}+T^{\prime}\left[w_{\epsilon}\right]\left(\phi_{\epsilon}\right) w_{\epsilon}^{2} .
$$

As $\epsilon \rightarrow 0$, we can compute easily that

$$
S_{\epsilon} \rightarrow S_{0}=\Delta \phi-\phi+2 w \phi-\frac{2 \int w \phi}{\int w^{2}} w^{2} .
$$

We now have

Lemma 6.1. For $\epsilon$ small,

$$
S_{\epsilon}: H_{s}^{2}\left(-\frac{l}{\epsilon}, \frac{l}{\epsilon}\right) \rightarrow L_{s}^{2}\left(-\frac{l}{\epsilon}, \frac{l}{\epsilon}\right)
$$

is one-to-one, onto, and thus invertible.

Proof: This follows from the fact that the operator $S_{0}$ is invertible in the space

$$
H_{s}^{2}(R)=\left\{u \in H^{2}(R) \mid u(y)=u(-y)\right\}
$$

From Lemma 6.1 and the fact that

$$
T\left[w_{\epsilon}\right](0)=(1+O(\epsilon)) \frac{1}{\xi_{0}}
$$

we see that (6.2) is solvable for $\left\|\phi_{\epsilon}\right\|_{H^{2}\left(-\frac{l}{\epsilon}, \frac{l}{\epsilon}\right)}$ small, by the contraction mapping principle.

\section{Appendix B. Computation of the matrix $\mathcal{B}$}

In this appendix we prove Proposition 2.1.

We first analyze problem (2.8) in this section. We use an indirect approach.

For $-1<x<x_{1}, \psi_{0}^{\prime \prime}=0$. Hence

$$
\psi_{0}(x)=\psi_{0}\left(x_{1}\right)=\eta_{1} .
$$

Similary, for $x_{i-1}<x<x_{i}, i=2, \ldots, N$

$$
\psi_{0}(x)=\eta_{i-1} \frac{x_{i}-x}{x_{i}-x_{i-1}}+\eta_{i} \frac{x-x_{i-1}}{x_{i}-x_{i-1}} .
$$


Hence

$$
\psi^{\prime}=\frac{N}{2}\left(\eta_{i}-\eta_{i-1}\right) \quad \text { for } x_{i-1}<x<x_{i} .
$$

Finally, for $x_{N}<x<1$, we have

$$
\psi_{0}^{\prime}(x)=0, \quad \psi_{0}(x)=\eta_{N}
$$

At $x_{i}, i=1, \ldots, N$, we have

$$
D\left[\psi^{\prime}\right]_{x_{i}}-\gamma \eta_{i}-2 c \int w \phi_{i}=0,
$$

where $\left[\psi^{\prime}\right]_{x_{i}}$ denotes the jump of $\psi^{\prime}$ at $x_{i}$. So at $x_{1}$, we have

$$
\frac{D N}{2}\left[\eta_{2}-\eta_{1}\right]=\gamma \eta_{1}+2 c \int w \phi_{1} \text {. }
$$

At $x_{i}, i=2, \ldots, N-1$ we have

$$
\frac{D N}{2}\left[\eta_{i+1}-2 \eta_{i}+\eta_{i-1}\right]=\gamma \eta_{i}+2 c \int w \phi_{i}
$$

At $x_{N}$, we have

$$
\frac{D N}{2}\left[0-\left(\eta_{N}-\eta_{N-1}\right)\right]=\gamma \eta_{N}+2 c \int w \phi_{N} .
$$

From (7.6), (7.7), and (7.8), we arrive at

$$
\eta=\left(\frac{D N}{2} K-\gamma I\right)^{-1} 2 c \int w \Phi
$$

which is exactly (2.12), where the matrix $K$ is given by (2.11) .

Hence

$$
\mathcal{B}=\gamma\left(\frac{D N}{2} K-\gamma I\right)^{-1}
$$

Since $K$ is a symmetric tridiagonal matrix, its eigenvalues of $K$ can be computed easily (see [9]):

$$
k_{j}=2\left(\cos \frac{\pi(j-1)}{N}-1\right), \quad j=1, \ldots, N
$$

and the eigenvectors of $K$ are

$$
\begin{gathered}
\mathbf{q}_{1}=\frac{1}{\sqrt{N}}(1, \ldots, 1)^{T}, \\
\mathbf{q}=\left(q_{l, 1}, \ldots, q_{l, N}\right), \quad l=2, \ldots, N, \\
q_{l, j}=\frac{1}{\sqrt{N}} \cos \left(\frac{\pi(j-1)}{N}\left(l-\frac{1}{2}\right)\right), \quad j=2, \ldots, N, \quad l=1, \ldots, N .
\end{gathered}
$$


Hence the eigenvalues of $\mathcal{B}$ are

$$
b_{j}=\gamma\left(\frac{D N}{2} k_{j}-\gamma\right)^{-1}, \quad j=1, \ldots, N
$$

\section{Appendix C: Computation of the eigenvalues of $\mathcal{M}$}

First, let us compute $G_{D}(x, z)$ - Green's function -

$$
\begin{cases}D G_{D}^{\prime \prime}(x, z)+\frac{1}{2}-\delta_{z}=0 & \text { in }(-1,1), \\ \int_{-1}^{1} G_{D}(x, z) d x=0, G_{D}^{\prime}(-1, z)=G_{D}^{\prime}(1, z)=0, & \end{cases}
$$

which was introduced in (3.15). It is easy to calculate that

$$
G_{D}(x, z)= \begin{cases}\frac{1}{D}\left[\frac{1}{3}-\frac{(x+1)^{2}}{4}-\frac{(1-z)^{2}}{4}\right], & -1<x \leq z, \\ \frac{1}{D}\left[\frac{1}{3}-\frac{(z+1)^{2}}{4}-\frac{(1-x)^{2}}{4}\right], & z \leq x<1 .\end{cases}
$$

We decompose

$$
G_{D}(x, z)=\frac{1}{2 D}|x-z|+H_{D}(x, z) .
$$

By simple computations,

$$
H_{D}(x, z)=\frac{1}{2 D}\left[-\frac{1}{3}-\frac{x^{2}}{2}-\frac{z^{2}}{2}\right]
$$

For $x \neq z$ we calculate

$$
\nabla_{x} \nabla_{z} G_{D}(x, z)=0, \quad \nabla_{x} G_{D}(x, z)= \begin{cases}-\frac{x+1}{2 D} & \text { if } x \leq z \\ -\frac{x-1}{2 D} & \text { if } z \leq x\end{cases}
$$

We further have

$$
\left.\nabla_{x} G_{D}(x, z)\right|_{x=z}=\left.\nabla_{x} H_{D}(x, z)\right|_{x=z}=-\frac{z}{2 D}
$$

Let $x_{j}=-1+\frac{2 j-1}{N}$. So we obtain

$$
\nabla \mathcal{G}_{D}=\left(c_{i j}\right)\left(-\frac{1}{2 D}\right),
$$

where

$$
c_{i j}=\left\{\begin{array}{cc}
x_{1}+1 & i<j \\
x_{i}-1 & i>j \\
x_{i} & i=j
\end{array}\right.
$$

We need to compute the eigenvalues of

$$
\mathcal{M}=-\frac{N}{2 D} I-\gamma \nabla \mathcal{G}_{D}\left(I+\gamma \mathcal{G}_{D}\right)^{-1}\left(\nabla \mathcal{G}_{D}\right)^{T}
$$




$$
=-\frac{N}{2 D} I-\gamma\left(\frac{1}{2 D}\right)^{2} C\left(I+\gamma \mathcal{G}_{D}\right)^{-1} C^{T}
$$

To this end, we introduce two matrices which will play very important roles. First, let us denote

$$
\mathbf{P}=\left(\mathbf{q}_{1}, \ldots, \mathbf{q}_{N}\right)
$$

where

$$
\left\{\begin{array}{c}
\mathbf{q}_{1}=\frac{1}{\sqrt{N}}(1, \ldots, 1), \\
\mathbf{q}_{l}=\left(q_{l, 1}, \ldots, q_{l, N}, \quad l=2, \ldots, N\right. \\
q_{l, j}=\sqrt{\frac{2}{N}} \cos \left(\frac{\pi(j-1)}{N}\left(l-\frac{1}{2}\right)\right), \quad j=2, \ldots, N .
\end{array}\right.
$$

(Note that $\mathbf{P}$ consists of the eigenvectors of $K$ in Appendix B.)

Similarly, we define

$$
\mathbf{Q}=\left(\mathbf{v}_{1}, \ldots, \mathbf{v}_{N}\right)
$$

where

$$
\left\{\begin{array}{c}
\mathbf{v}_{1}=\frac{1}{\sqrt{N}}\left(1,-1,1, \ldots,(-1)^{N+1}\right), \\
\mathbf{v}_{l}=\left(v_{l, 1}, \ldots, v_{l, N}, \quad l=2, \ldots, N\right. \\
v_{l, j}=\sqrt{\frac{2}{N}} \sin \left(\frac{\pi(j-1)}{N}\left(l-\frac{1}{2}\right)\right), \quad j=2, \ldots, N .
\end{array}\right.
$$

We now make a few claims:

\section{Claim I:}

$$
\mathbf{P}^{-1} \mathcal{G}_{D} \mathbf{P}=\left(\begin{array}{ccc}
\lambda_{1} & & \\
& \ddots & \\
& & \lambda_{N}
\end{array}\right)
$$

where

$$
\lambda_{1}=-\frac{1}{6 D N}, \quad \lambda_{j}=-\frac{1}{2 D N \sin ^{2}\left(\frac{\pi(j-1)}{2 N}\right)}, \quad j=2, \ldots, N .
$$

\section{Claim II:}

$$
\mathrm{Q} \nabla \mathcal{G}_{D} \mathbf{P}=\left(\begin{array}{ccc}
\nu_{1} & & \\
& \ddots & \\
& & \nu_{N}
\end{array}\right)
$$

where

$$
\nu_{1}=0, \quad \nu_{j}=\frac{1}{2 D \tan \frac{\pi(j-1)}{2 N}}, \quad j=2, \ldots, N
$$


We shall verify these two claims at the end of the appendix. Let us suppose the above two claims are true. Then the eigenvalues of $\mathcal{M}$ are given by

$$
m_{j}=-\frac{N}{2 D}-\gamma \nu_{j}^{2}\left(1+\gamma \lambda_{j}\right)^{-1} .
$$

So $m_{1}=-\frac{N}{2 D}$ and for $j=2, \ldots, N$,

$$
m_{j}=-\frac{N}{2 D}-\gamma\left(\frac{1}{2 D}\right)^{2} \frac{1}{\tan ^{2} \frac{\theta_{j}}{2}}\left(1-\frac{\gamma}{2 D N \sin ^{2} \frac{\theta_{j}}{2}}\right)^{-1},
$$

which proves Lemma 3.2.

Finally we prove Claim I and Claim II.

Proof of Claim I: Let $\theta \neq 0$. We recall the following formulas:

$$
\begin{gathered}
\sum_{l=1}^{N} \cos \theta\left(l-\frac{1}{2}\right)=\frac{\sin \theta N}{2 \sin \frac{\theta}{2}}, \\
\sum_{l=1}^{N}\left(l-\frac{1}{2}\right) \cos \theta\left(l-\frac{1}{2}\right)=\frac{N \sin \frac{\theta}{2} \sin N \theta-\frac{1}{2}(1-\cos \theta N) \cos \frac{\theta}{2}}{2 \sin ^{2} \frac{\theta}{2}} \\
\sum_{l=1}^{N}\left(l-\frac{1}{2}\right)^{2} \cos \theta\left(l-\frac{1}{2}\right)=-\frac{N}{2}\left(\frac{\cos N \theta}{\sin \frac{\theta}{2}}\right)_{\theta}+\left(\frac{\cos \frac{\theta}{2} \sin N \theta}{4 \sin ^{2} \frac{\theta}{2}}\right)_{\theta} .
\end{gathered}
$$

To prove Claim I, all we need to check is

$$
\sum_{l=1}^{N} G_{D}\left(x_{i}, x_{l}\right) q_{l, j}=\lambda_{j} q_{i, j} .
$$

When $j=1$, we have that

$$
\begin{gathered}
2 D \sum_{l=1}^{N} G_{D}\left(x_{i}, x_{l}\right)=\left(-\frac{1}{3}-\frac{x_{i}^{2}}{2}-\frac{1}{2}\right) N+\frac{2}{N} \sum_{l=1}^{N}\left(l-\frac{1}{2}\right) \\
-\frac{2}{N^{2}} \sum_{l=1}^{N}\left(l-\frac{1}{2}\right)^{2}+\frac{1}{2 D} \frac{2}{N} \sum_{l=1}^{N}|l-i| \\
=\left(-\frac{1}{3}-\frac{x_{i}^{2}}{2}\right) N+\sum_{l=1}^{N}|l-i| \frac{2}{N}-\frac{1}{2} \sum_{l=1}^{N} x_{l}^{2} \\
=2 D \lambda_{1},
\end{gathered}
$$

where $\lambda_{1}$ is given by (8.11).

For $j=2, \ldots, N$, we need to check that

$$
\sum_{l=1}^{N} G_{D}\left(x_{i}, x_{l}\right) \cos \left(\theta_{j}\left(l-\frac{1}{2}\right)\right)=\lambda_{j} \cos \left(\theta_{j}\left(i-\frac{1}{2}\right)\right),
$$


where

Note that

$$
\theta_{j}=\frac{\pi(j-1)}{N}
$$

$$
\begin{gathered}
2 D G_{D}\left(x_{i}, x_{l}\right)=\left|x_{i}-x_{l}\right|-\frac{1}{3}-\frac{x_{i}^{2}}{2}-\frac{x_{l}^{2}}{2} \\
=-\frac{1}{3}-\frac{x_{i}^{2}}{2}-\frac{1}{2}+\frac{2\left(l-\frac{1}{2}\right)}{N}-\frac{2\left(l-\frac{1}{2}\right)^{2}}{N^{2}}+\left|x_{i}-x_{l}\right| .
\end{gathered}
$$

Since

we have that

$$
\sum_{l=1}^{N} \cos \theta_{j}\left(l-\frac{1}{2}\right)=\frac{\sin N \theta_{j}}{2 \sin \frac{\theta_{j}}{2}}=0,
$$

$$
\begin{aligned}
& 2 D \sum_{l=1}^{N} G_{D}\left(x_{i}, x_{l}\right) \cos \left(\theta_{j}\left(l-\frac{1}{2}\right)\right) \\
& =\frac{2}{N} \sum_{l=1}^{N}\left(l-\frac{1}{2}\right) \cos \left(\theta_{j}\left(l-\frac{1}{2}\right)\right)-\frac{2}{N^{2}} \sum_{l=1}^{N}\left(l-\frac{1}{2}\right)^{2} \cos \theta_{j}\left(l-\frac{1}{2}\right) \\
& +\frac{2}{N} \sum_{l=1}^{N}(l-i) \cos \theta_{j}\left(l-\frac{1}{2}\right)+\frac{4}{N} \sum_{l=1}^{i}(i-l) \cos \theta_{j}\left(l-\frac{1}{2}\right) \\
& =\frac{4}{N}\left(\frac{\left(\cos N \theta_{j}-1\right) \cos \frac{\theta_{j}}{2}}{4 \sin ^{2} \frac{\theta_{j}}{2}}\right) \\
& -\frac{2}{N^{2}}\left(-\frac{N}{4} \frac{-\cos N \theta_{j} \cos \frac{\theta_{j}}{2}}{\sin ^{2} \frac{\theta_{j}}{2}}+\frac{N \cos \frac{\theta_{j}}{2} \cos N \theta_{j} \sin ^{2} \frac{\theta_{j}}{2}}{4 \sin ^{4} \frac{\theta_{j}}{2}}\right) \\
& +\frac{4}{N}\left(\left(i-\frac{1}{2}\right) \sum_{l=1}^{i} \cos \theta_{j}\left(l-\frac{1}{2}\right)-\sum_{l=1}^{i}\left(l-\frac{1}{2}\right) \cos \theta_{j}\left(l-\frac{1}{2}\right)\right) \\
& =\frac{4}{N}\left[\left(i-\frac{1}{2}\right) \frac{\sin \theta_{j} i}{2 \sin \frac{\theta_{j}}{2}}-\frac{i \sin \frac{\theta_{j}}{2} \sin \theta_{j} i-\left(1-\cos \theta_{j} i\right) \frac{1}{2} \cos \frac{\theta_{j}}{2}}{2 \sin ^{2} \frac{\theta_{j}}{2}}\right]-\frac{\cos \frac{\theta_{j}}{2}}{N \sin ^{2} \frac{\theta_{j}}{2}} \\
& =-\frac{\cos \theta_{j}\left(i-\frac{1}{2}\right)}{N \sin ^{2} \frac{\theta_{j}}{2}}
\end{aligned}
$$

Therefore we obtain that

$$
\lambda_{j}=-\frac{1}{2 D N \sin ^{2} \frac{\pi(j-1)}{2 N}}, \quad j=2, \ldots, N,
$$

which proves Claim I.

Next we prove Claim II. 


\section{Proof of Claim II:}

We need to show that

$$
\left(-\frac{1}{2 D}\right) \sum_{l=1}^{N} c_{i l} \cos \left(\frac{\pi(j-1)}{N}\left(l-\frac{1}{2}\right)\right)=\nu_{j} \sin \left(\frac{\pi(j-1)}{N}\left(i-\frac{1}{2}\right)\right) .
$$

For $j=1$ we calculate

$$
\sum_{l=1}^{N} c_{i l}=\sum_{l=1}^{N} x_{i}+(N-i)=x_{i} N+N-2 i-1=0
$$

So we get

$$
\nu_{1}=0 \text {. }
$$

For $j \geq 2$, we consider

$$
\begin{aligned}
& \sum_{l=1}^{N} c_{i l} \cos \left(\frac{\pi(j-1)}{N}\left(l-\frac{1}{2}\right)\right) \\
& =\sum_{l=1}^{N} x_{i} \cos \left(\frac{\pi(j-1)}{N}\left(l-\frac{1}{2}\right)\right) \\
& +\sum_{l=1}^{i-1} \cos \left(\theta_{j}\left(l-\frac{1}{2}\right)\right)+\sum_{l=i+1}^{N} \cos \left(\theta_{j}\left(l-\frac{1}{2}\right)\right) \\
& =-\frac{\sin \theta_{j}(i-1)}{2 \sin \frac{\theta_{j}}{2}}+\frac{1}{2 \sin \frac{\theta_{j}}{2}}\left(\sin N \theta_{j}-\sin i \theta_{j}\right) \\
& =-\frac{\sin \theta_{j}(i-1)+\sin i \theta_{j}}{2 \sin \frac{\theta_{j}}{2}} \\
& =-\frac{2 \sin \theta_{j}\left(i-\frac{1}{2}\right) \cos \frac{\theta_{j}}{2}}{2 \sin \frac{\theta_{j}}{2}} \\
& =-\frac{1}{\tan \frac{\theta_{j}}{2}} \sin \theta_{j}\left(i-\frac{1}{2}\right) .
\end{aligned}
$$

Claim II is thus proved. 


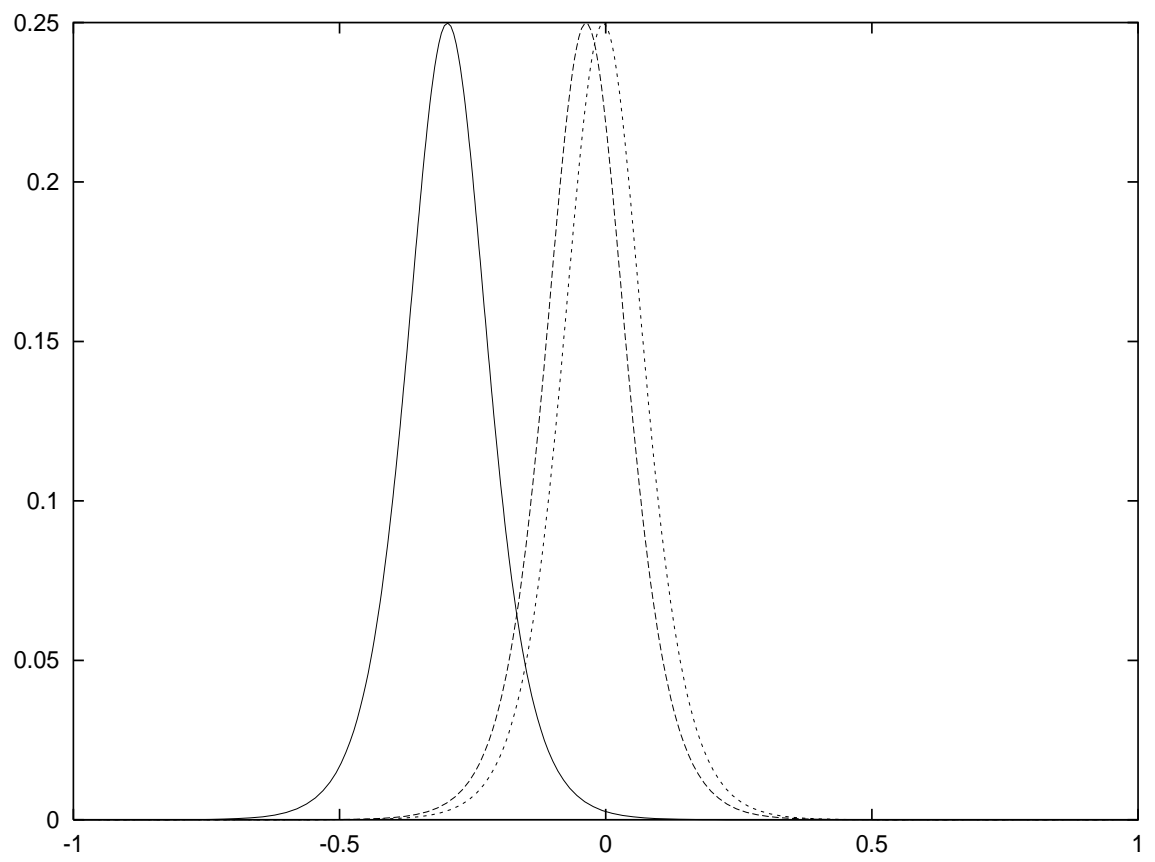

Figure 2. Dynamics of one-spike solutions. Here $D=5, \epsilon=$ 0.05 and the trajectories are at $\mathrm{t}=50, \mathrm{t}=2500$ and $\mathrm{t}=50000$. 


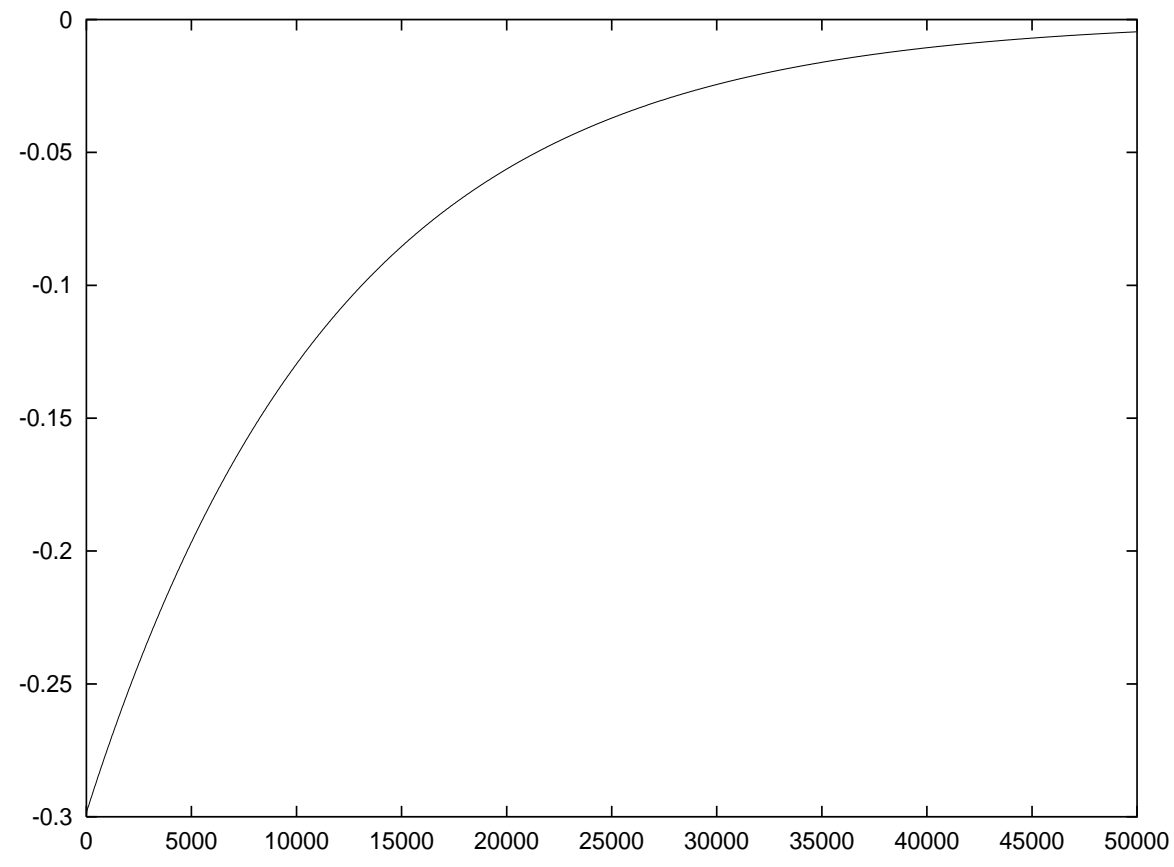

Figure 3 . Trajectory of the center $x_{0}(t)$. 


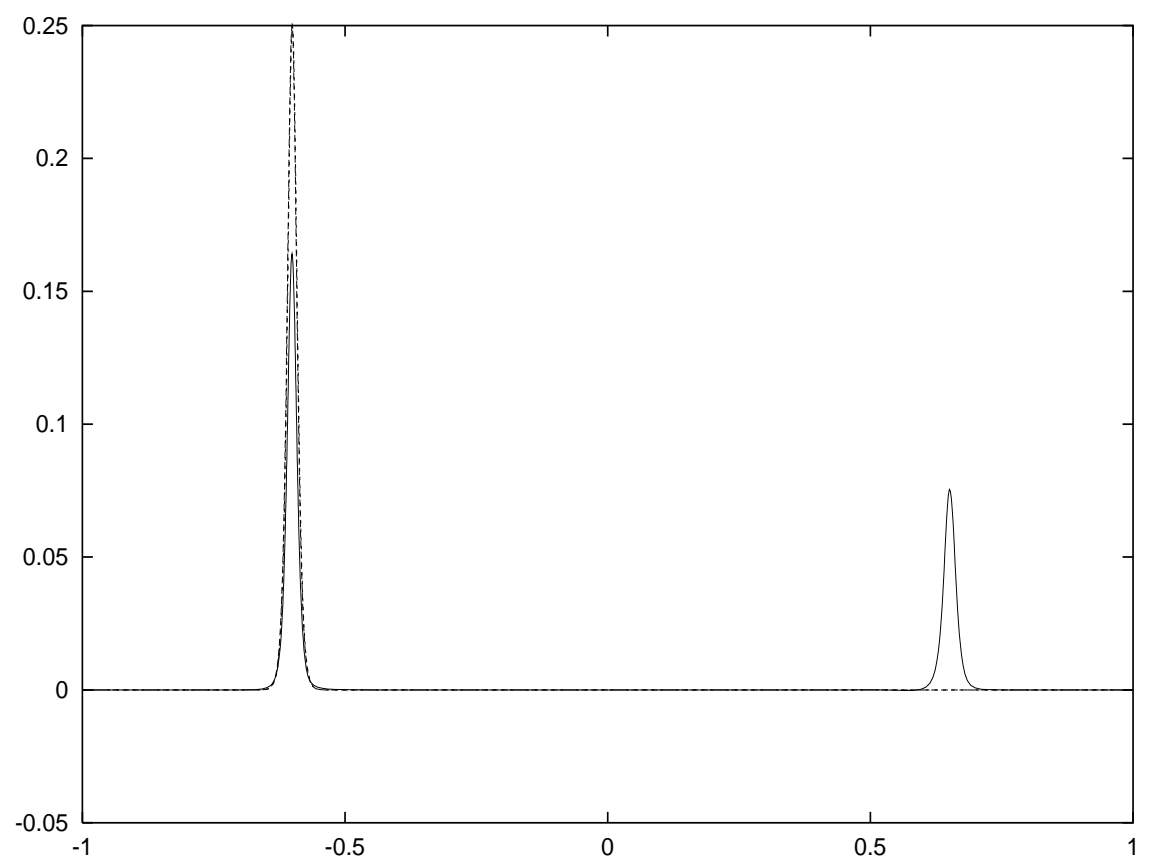

Figure 4. Unstable two-peaked solution above $D_{2}$. Here $D=$ $1>D_{2}, \epsilon=0.06$ and the trajectory is taken at $t=100$. 


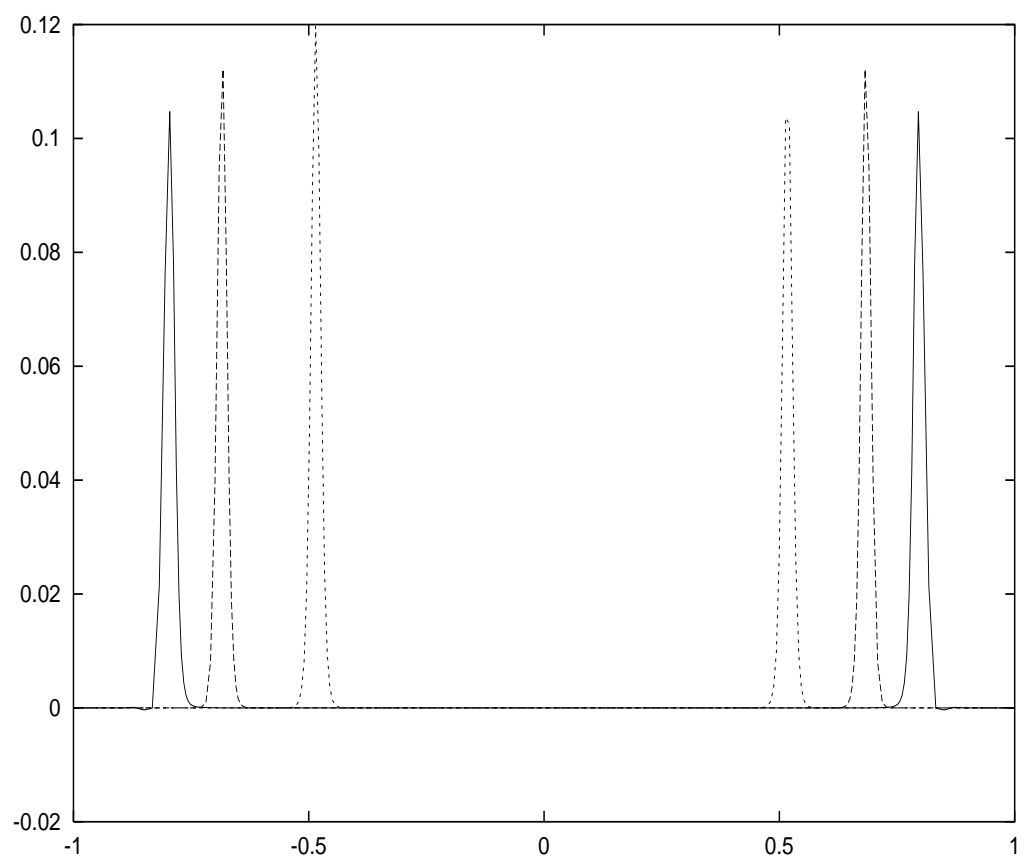

Figure 5. Stable two-peaked solution below $D_{2}$. Here $D=$ $0.08<D_{2}, \epsilon=0.06$ and the trajectories are taken at $t=$ 10,100 and 1000. 


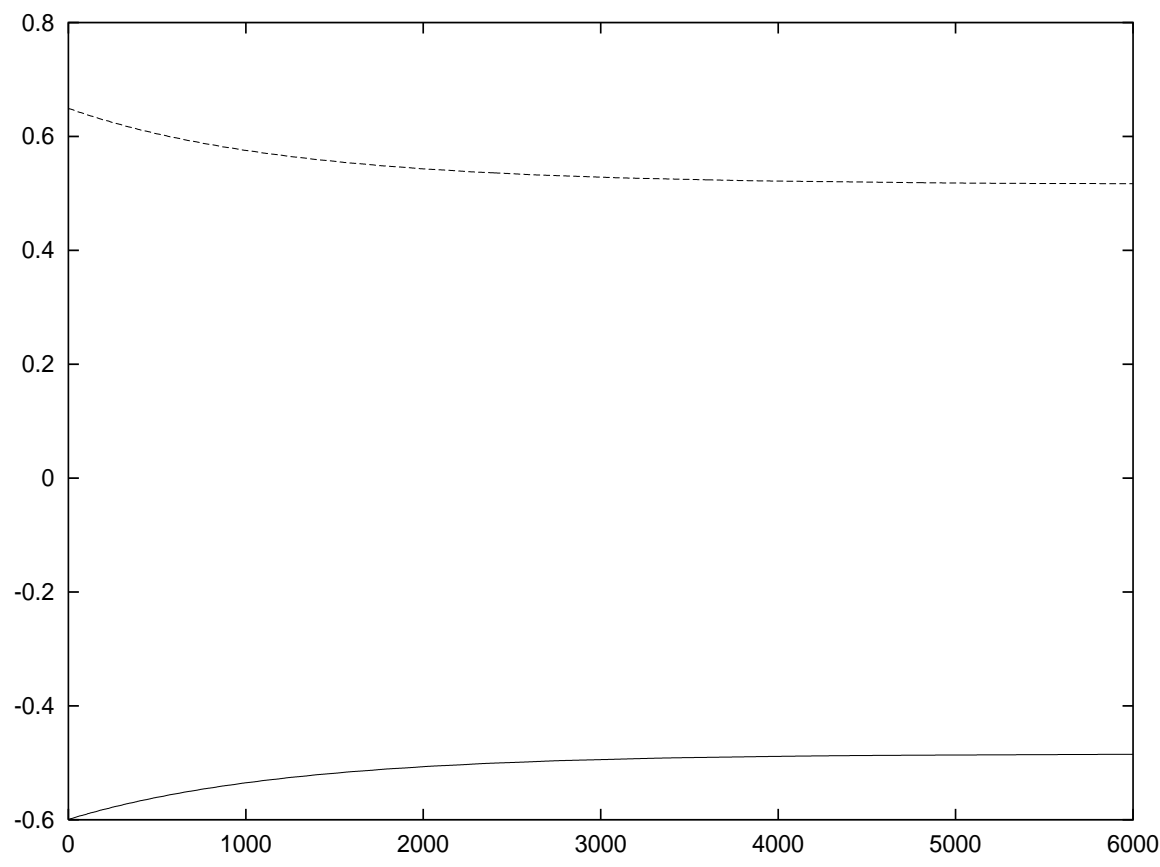

Figure 6. Trajectories of the two centers. Here $D=0.008<$ $D_{2}, \epsilon=0.06$. 


\section{REFERENCES}

[1] E.N. Dancer, On stability and Hopf bifurcations for chemotaxis systems, Meth. Appl. Anal. 8 (2001), 245-256.

[2] A. Doelman, R. A. Gardner, and T. J. Kaper, Stability analysis of singular patterns in the 1D gray-Scott model: a matched asymptotics approach, Phys. D 122 (1998), $1-36$.

[3] A. Doelman, R. A. Gardner, and T. J. Kaper, Large stable pulse solutions in reaction-diffusion equations, Indiana Univ. Math. J. 50 (2001), 443-507.

[4] A. Doelman, T. J. Kaper, and P. A. Zegeling, Pattern formation in the onedimensional Gray-Scott model, Nonlinearity 10 (1997), 523-563.

[5] A. Gierer and H. Meinhardt, A theory of biological pattern formation, Kybernetik (Berlin) 12 (1972), 30-39.

[6] D. Gilbarg and N. S. Trudinger, Elliptic Partial Differential Equations of Second Order, 2nd edition, Springer, Berlin, 1983.

[7] C. Gui and J. Wei, Multiple interior peak solutions for some singular perturbation problems, J. Differential Equations 158 (1999), 1-27.

[8] C. Gui, J. Wei and M. Winter, Multiple boundary peak solutions for some singularly perturbed Neumann problems, Ann. Inst. H. Poincaré Anal. Non Linéaire 17 (2000), 47-82.

[9] D. Iron, M. J. Ward, and J. Wei, The stability of spike solutions to the onedimensional Gierer-Meinhardt model, Phys. D 150 (2001), 25-62.

[10] D. L. Benson, P. K. Maini, and J. A. Sherratt, Unravelling the Turing bifurcation using spatially varying diffusion coefficients, J. Math. Biol. 37 (1998), 381-417.

[11] W.-M. Ni, Diffusion, cross-diffusion, and their spike-layer steady states, Notices Amer. Math. Soc. 45 (1998), 9-18.

[12] W.-M. Ni and I. Takagi, On the shape of least energy solution to a semilinear Neumann problem, Comm. Pure Appl. Math. 41 (1991), 819-851.

[13] W.-M. Ni and I. Takagi, Locating the peaks of least energy solutions to a semilinear Neumann problem, Duke Math. J. 70 (1993), 247-281.

[14] W.-M. Ni, I. Takagi and E. Yanagida, Tohoku Math. J., to appear.

[15] Y. Nishiura, Coexistence of infinitely many stable solutions to reaction-diffusion equation in the singular limit, in Dynamics reported: Expositions in Dynamical Systems, Volume 3, Editors: C. K. R. T. Jones, U. Kirchgraber, Springer Verlag, New York, (1995).

[16] J. Schnakenberg, Simple chemical reaction systems with limit cycle behaviour, $J$. Theoret. Biol. 81 (1979), 389-400.

[17] I. Takagi, Point-condensation for a reaction-diffusion system, J. Differential Equations 61 (1986), 208-249.

[18] A. M. Turing, The chemical basis of morphogenesis, Phil. Trans. Roy. Soc. Lond. B 237 (1952), 37-72.

[19] M.J. Ward and J. Wei, Asymmetric spike patterns for the one-dimensional GiererMeinhardt model: equilibria and stability, Europ. J. Appl. Math. 13 (2002), 283-320.

[20] J. Wei, On the boundary spike layer solutions of singularly perturbed semilinear Neumann problem, J. Diffential Equations 134 (1997), 104-133.

[21] J. Wei, On the interior spike layer solutions of singularly perturbed semilinear Neumann problem, Tohoku Math. J. 50 (1998), 159-178.

[22] J. Wei, On the interior spike layer solutions for some singular perturbation problems, Proc. Royal Soc. Edinburgh, Section A (Mathematics) 128 (1998), 849-874. 
[23] J. Wei, On single interior spike solutions of Gierer-Meinhardt system: uniqueness and spectrum estimates, Europ. J. Appl. Math. 10 (1999), 353-378.

[24] M. J. Ward and J. Wei, The existence and stability of asymmetric spike patterns for the Schnakenberg model, Stud. Appl. Math. 109 (2002), 229-264.

[25] J. Wei and M. Winter, On the two-dimensional Gierer-Meinhardt system with strong coupling, SIAM J. Math. Anal. 30 (1999), 1241-1263.

[26] J. Wei and M. Winter, Spikes for Gierer-Meinhardt system in two dimensions: The strong coupling case, J. Differential Equations 178 (2002), 478-518.

[27] J. Wei and M. Winter, Spikes for the two-dimensional Gierer-Meinhardt system: The weak coupling case, J. Nonlinear Sci. 11 (2001), 415-458.

[28] J. Wei and M. Winter, Existence and stability analysis of multiple-peaked solutions, submitted.

Department of Mathematics, University of California at Irvine, Irvine, CA 92697-3875, USA

E-mail address: diron@math.uci.edu

Department of Mathematics, The Chinese University of Hong Kong, Shatin, HONG KONG

E-mail address: wei@math.cuhk.edu.hk

Fachbereich Mathematik, Universität Stuttgart, Pfaffenwaldring 57, D70569 Stuttgart, Germany

E-mail address: winter@mathematik.uni-stuttgart.de 